Supporting Data for

\title{
New chromium(III) complexes for photochemical nitric oxide generation from coordinated nitrite. The synthesis and photochemistry of macrocyclic complexes with pendant chromophores, trans- $\left[\mathrm{Cr}(\mathrm{L})(\mathrm{ONO})_{2}\right] \mathrm{BF}_{4}$
}

\author{
Frank DeRosa, Xianhui Bu, Peter C. Ford* \\ Department of Chemistry and Biochemistry, University of California, Santa Barbara, \\ Santa Barbara, CA 93106-9510, e.mail: ford@chem.ucsb.edu
}

\section{List of Figures and Tables}

Figure S1. Spectrum of trans- $\left[\mathrm{Cr}(\right.$ cyclam $\left.)(\mathrm{ONO})_{2}\right] \mathrm{BF}_{4}(\mathbf{I})(7.8 \mathrm{mM})$ in aqueous solution. Inset: same data for $500-750 \mathrm{~nm}$ in an 25 -fold expanded view.

Figure S2 Top: Difference spectra after photolysis of an aqueous solution of trans- $\left[\mathrm{Cr}(\mathrm{mbc})(\mathrm{ONO})_{2}\right] \mathrm{BF}_{4}(\mathbf{I I})(1 \mathrm{mM})$ under aerated conditions at ambient temperature over a period of $48 \mathrm{~h}$. $\lambda_{\text {irr }}=365 \mathrm{~nm}$. (-) uses $\mathrm{t}$ $=0$ as the baseline. $(\diamond)$ uses $\mathrm{t}=1 \mathrm{~h}$ as the baseline. Bottom: Difference spectra after photolysis of an aqueous solution of trans$\left[\mathrm{Cr}(\right.$ cyclam $\left.)(\mathrm{ONO})_{2}\right] \mathrm{BF}_{4}(\mathbf{I})(1 \mathrm{mM})$ under aerated conditions at ambient temperature over a period of $48 \mathrm{~h}$. $\lambda_{\text {irr }}=365 \mathrm{~nm}$. (-) uses $\mathrm{t}$ $=0$ as the baseline. $(\diamond)$ uses $\mathrm{t}=1 \mathrm{~h}$ as the baseline.

Figure S3 Mass spectra of photolysis products of trans- $\left[\mathrm{Cr}(\mathrm{mac})(\mathrm{ONO})_{2}\right] \mathrm{BF}_{4}$ (III) during the first two hours of photolysis $\left(\lambda_{\text {irr }}=365 \mathrm{~nm}\right)$.

Figure S4. Excitation spectra of dilute aqueous solutions of trans$\left[\mathrm{Cr}(\mathrm{mac})(\mathrm{ONO})_{2}\right] \mathrm{BF}_{4}(\mathrm{III})(50 \mu \mathrm{M})$ and its respective free ligand, mac $(50 \mu \mathrm{M}) . \lambda_{\text {mon }}=420 \mathrm{~nm}$.

Figure S5. Emission spectra of a dilute 1:1 acetonitrile: $\mathrm{H}_{2} \mathrm{O}(v / v)$ solutions of trans- $\left[\mathrm{Cr}(\mathrm{pbc})(\mathrm{ONO})_{2}\right] \mathrm{BF}_{4}(\mathbf{I V})(30 \mu \mathrm{M})$ and its respective free chromophore, 1-pyrenecarboxylic acid $(30 \mu \mathrm{M}) . \lambda_{\text {irr }}=340 \mathrm{~nm}$. 
Figure S6 Excitation spectra of dilute 1:1 acetonitrile: $\mathrm{H}_{2} \mathrm{O}(v / v)$ solutions of trans- $\left[\mathrm{Cr}(\mathrm{pbc})(\mathrm{ONO})_{2}\right] \mathrm{BF}_{4}(\mathbf{I} \mathbf{V})(30 \mu \mathrm{M})$ and its respective free chromophore, 1-pyrenecarboxylic acid $(30 \mu \mathrm{M}) . \lambda_{\text {mon }}=420 \mathrm{~nm}$.

Table S1. Infrared absorption data of chromium(III) nitrito complexes in $\mathrm{KBr}$.

Tables S2a-e, S3a-e, S4a-2 X-Ray Crystal Data 
Figure S1 Spectrum of trans-[Cr(cyclam $\left.)(\mathrm{ONO})_{2}\right] \mathrm{BF}_{4}(\mathbf{I})(7.8 \mathrm{mM})$ in aqueous solution. Inset: same data for $500-750 \mathrm{~nm}$ in an 25-fold expanded view.

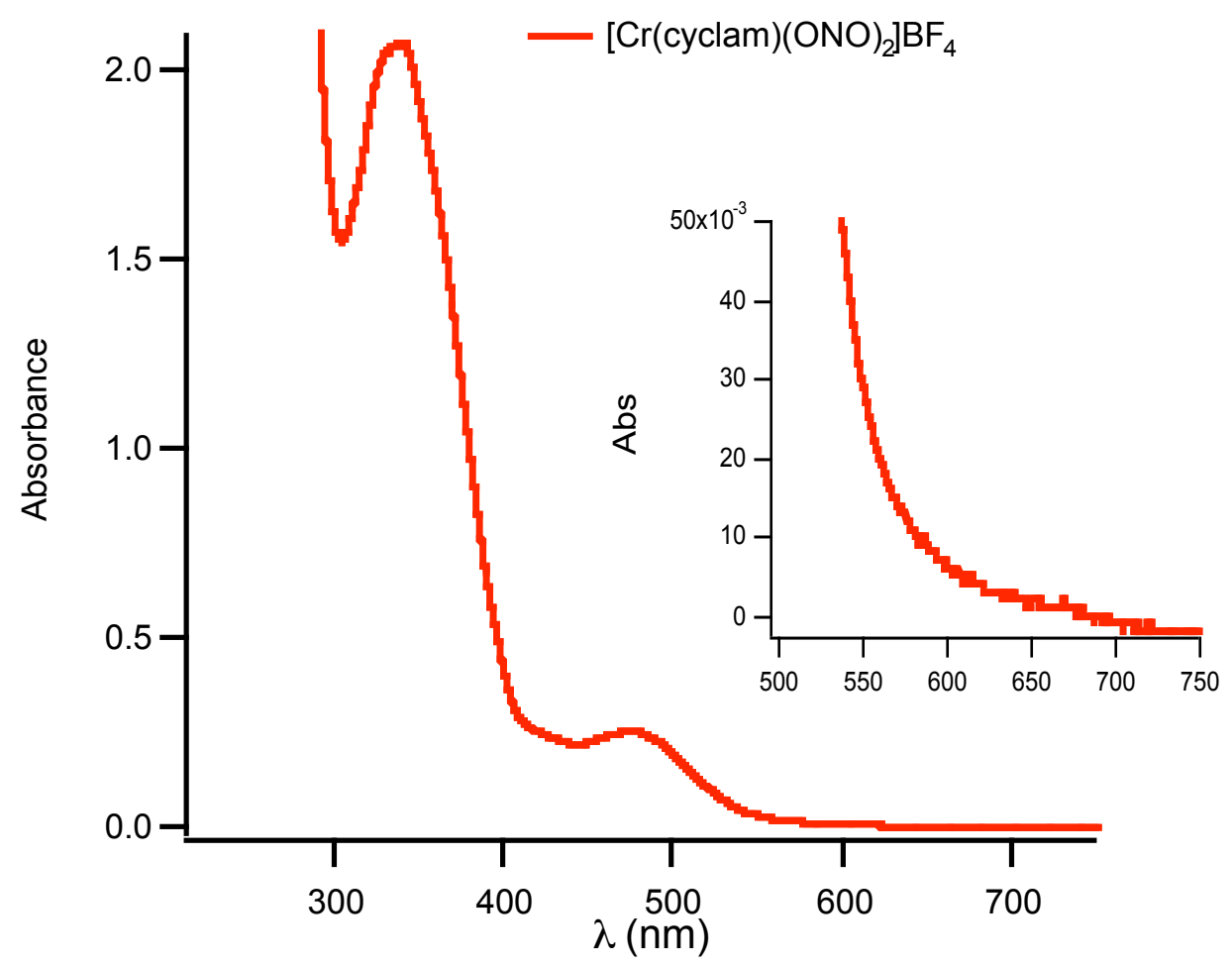


Figure S2 Top: Difference spectra after photolysis of an aqueous solution of trans- $\left[\mathrm{Cr}(\mathrm{mbc})(\mathrm{ONO})_{2}\right] \mathrm{BF}_{4}(\mathbf{I I})(1 \mathrm{mM})$ under aerated conditions at ambient temperature over a period of $48 \mathrm{~h}$. $\lambda_{\text {irr }}=365 \mathrm{~nm}$. (-) uses $\mathrm{t}$ $=0$ as the baseline. $(\diamond)$ uses $\mathrm{t}=1 \mathrm{~h}$ as the baseline. Bottom: Difference spectra after photolysis of an aqueous solution of trans$\left[\mathrm{Cr}(\right.$ cyclam $\left.)(\mathrm{ONO})_{2}\right] \mathrm{BF}_{4}(\mathbf{I})(1 \mathrm{mM})$ under aerated conditions at ambient temperature over a period of $48 \mathrm{~h}$. $\lambda_{\text {irr }}=365 \mathrm{~nm}$. (-) uses $\mathrm{t}$ $=0$ as the baseline. $(\diamond)$ uses $\mathrm{t}=1 \mathrm{~h}$ as the baseline.
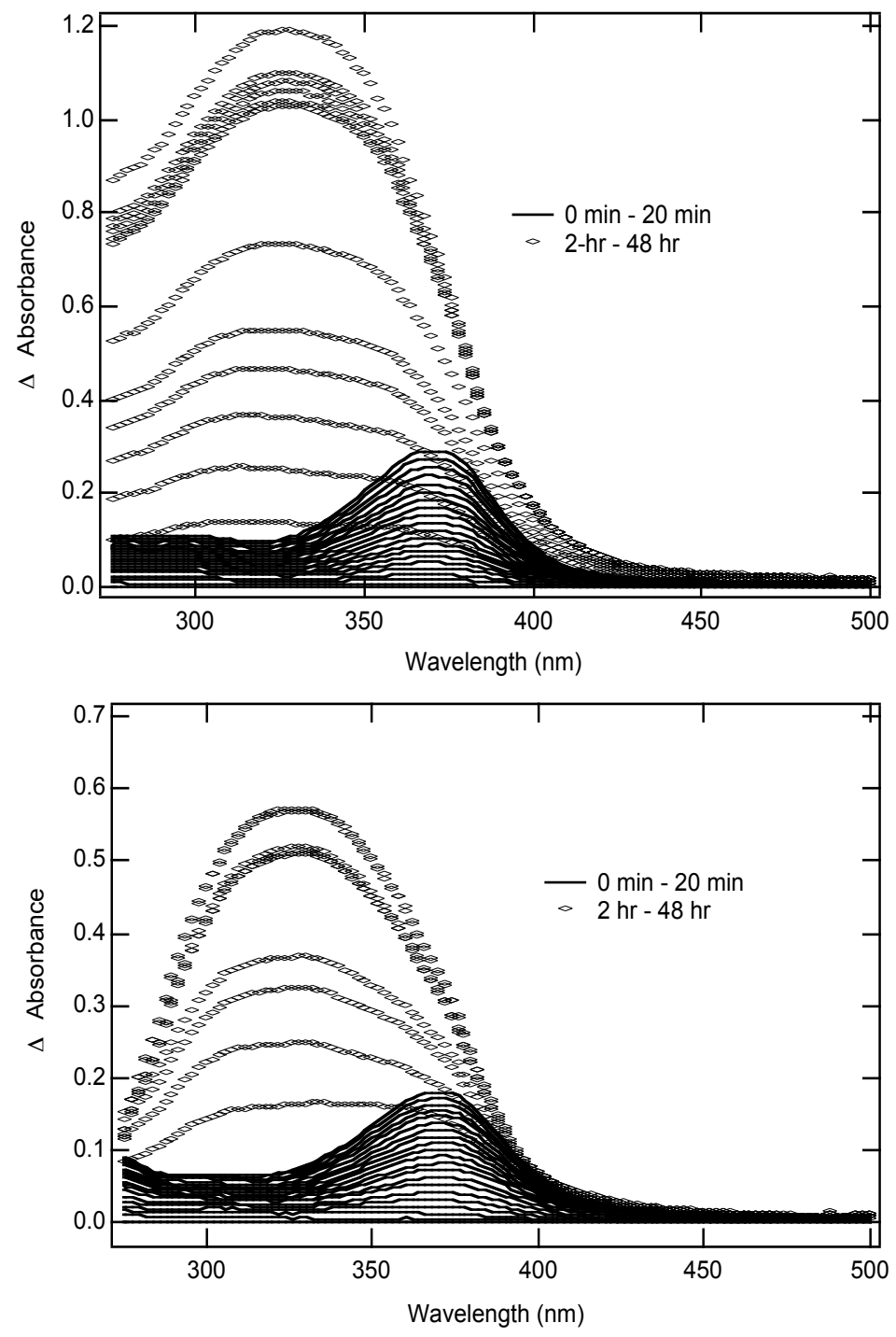
Figure S3. Mass spectra of photolysis products of trans-[Cr(mac)(ONO) $\left.)_{2}\right] \mathrm{BF}_{4}$ (III) during the first two hours of photolysis $\left(\lambda_{\text {irr }}=365 \mathrm{~nm}\right)$.

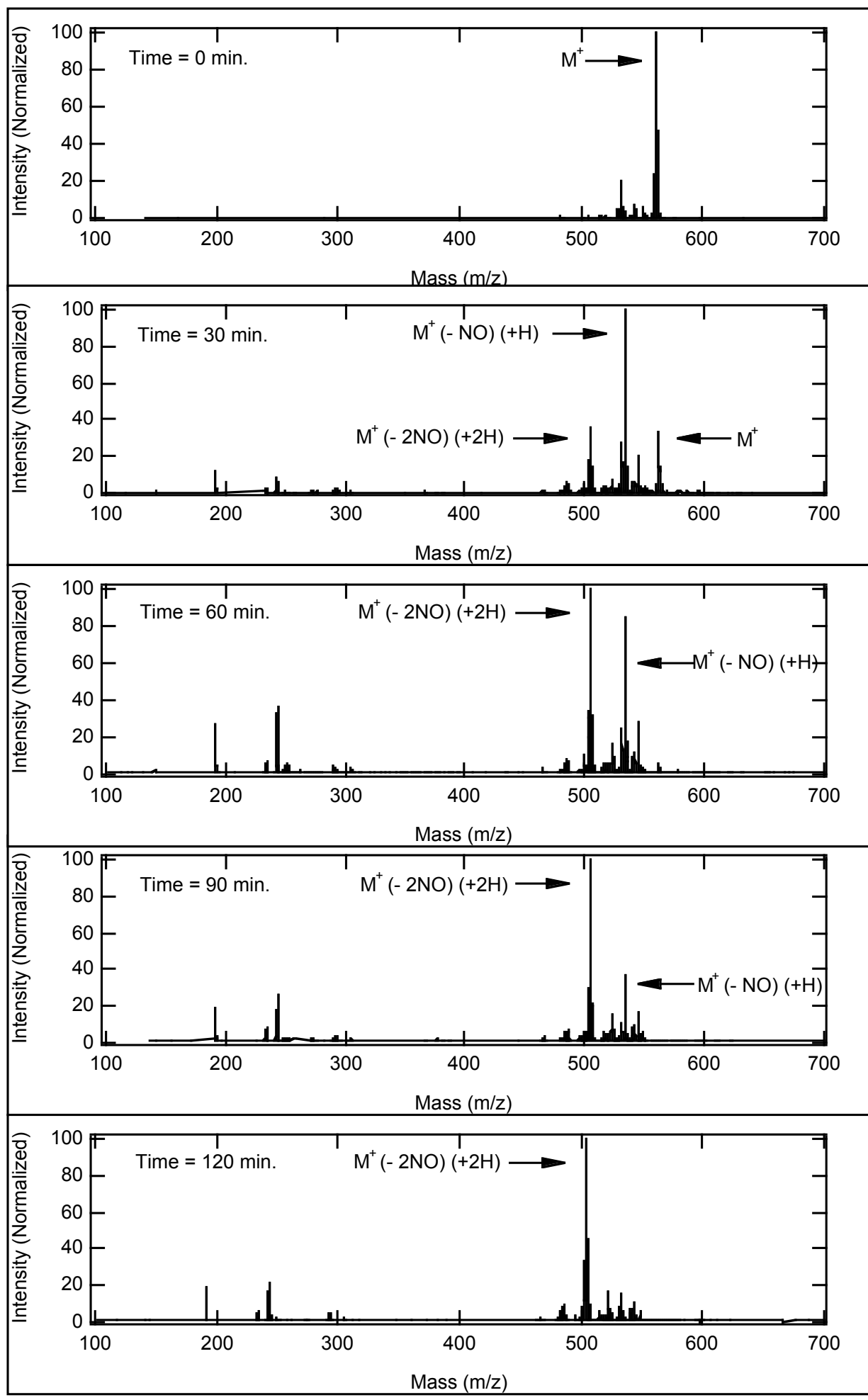


Figure S4. Excitation spectra of dilute aqueous solutions of trans-

$\left[\mathrm{Cr}(\mathrm{mac})(\mathrm{ONO})_{2}\right] \mathrm{BF}_{4}(\mathbf{I I I})(50 \mu \mathrm{M})$ and its respective free ligand, mac $(50 \mu \mathrm{M}) . \lambda_{\text {mon }}=420 \mathrm{~nm}$.

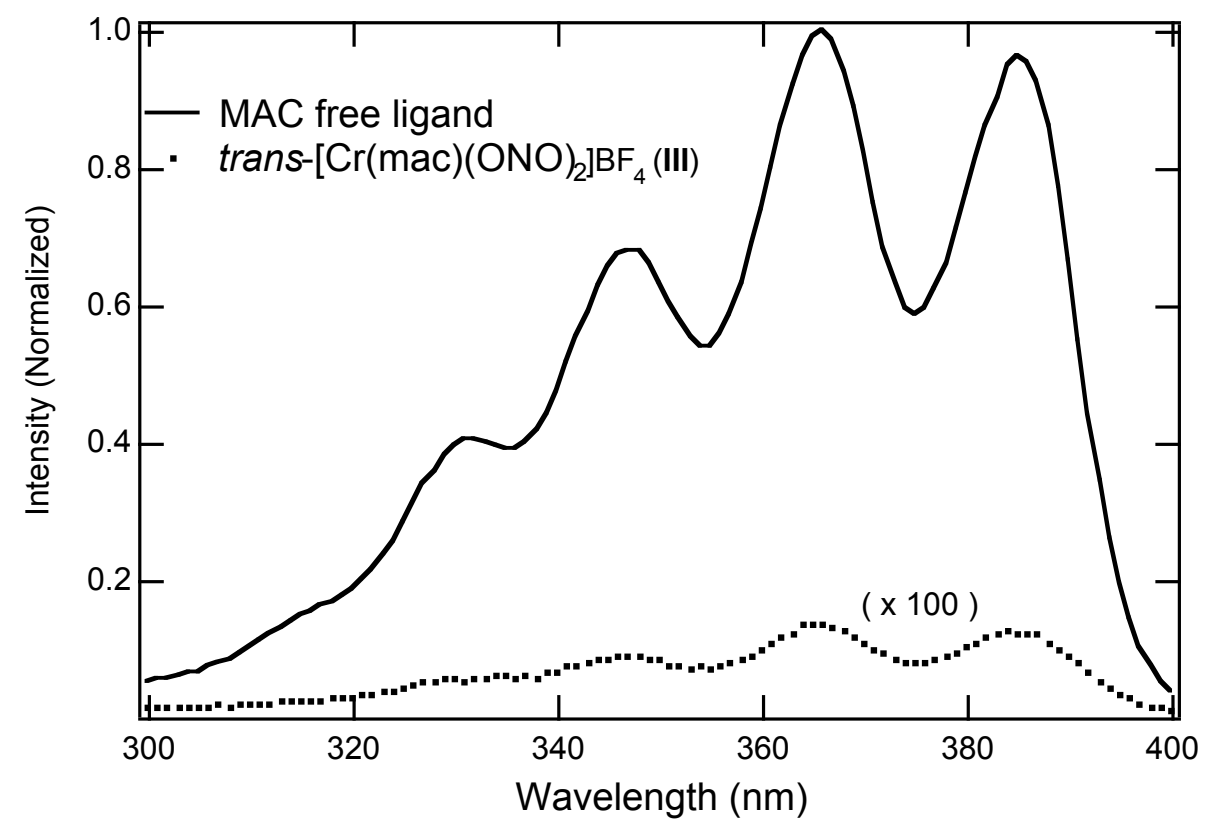


Figure S5. Emission spectra of a dilute 1:1 acetonitrile: $\mathrm{H}_{2} \mathrm{O}(v / v)$ solutions of trans- $\left[\mathrm{Cr}(\mathrm{pbc})(\mathrm{ONO})_{2}\right] \mathrm{BF}_{4}(\mathbf{I V})(30 \mu \mathrm{M})$ and its respective free chromophore, 1-pyrenecarboxylic acid $(30 \mu \mathrm{M}) . \lambda_{\text {irr }}=340 \mathrm{~nm}$.

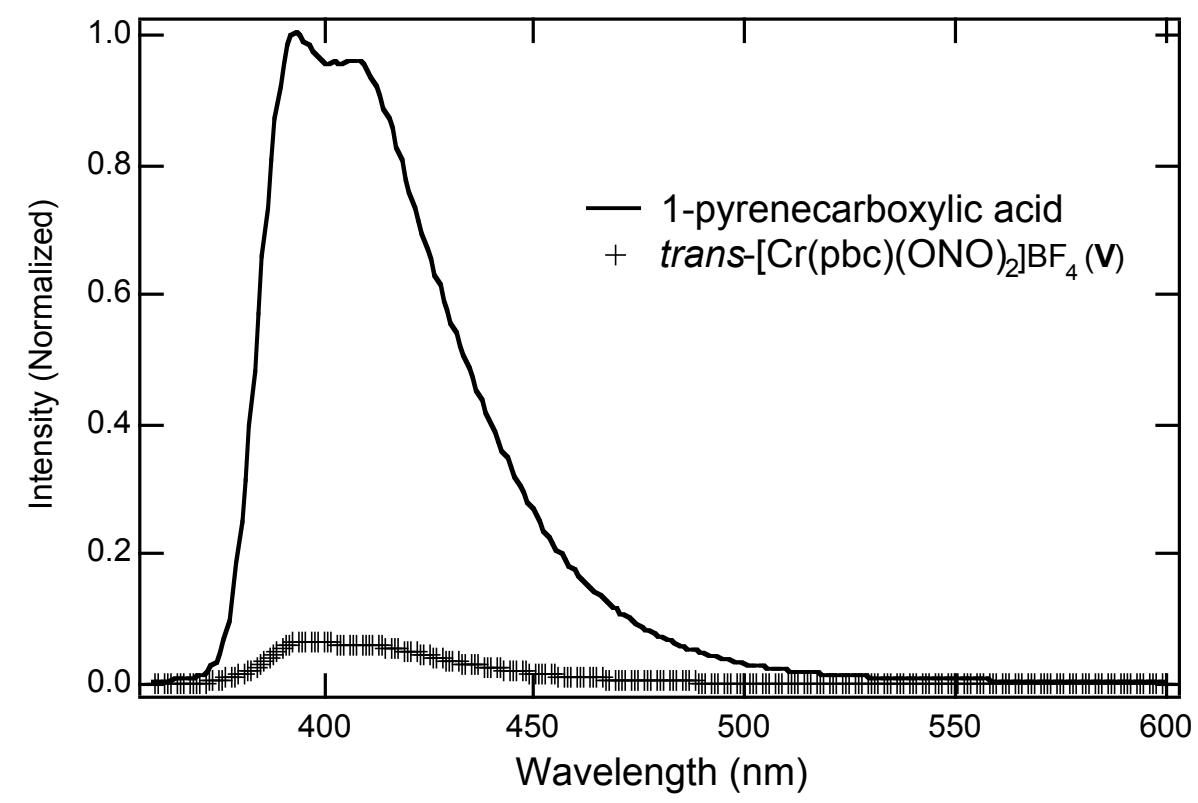


Figure S6 Excitation spectra of dilute 1:1 acetonitrile: $\mathrm{H}_{2} \mathrm{O}(v / v)$ solutions of trans $-\left[\mathrm{Cr}(\mathrm{pbc})(\mathrm{ONO})_{2}\right] \mathrm{BF}_{4}(\mathbf{I ~ V})(30 \mu \mathrm{M})$ and its respective free chromophore, 1-pyrenecarboxylic acid $(30 \mu \mathrm{M}) . \quad \lambda_{\text {mon }}=420 \mathrm{~nm}$.

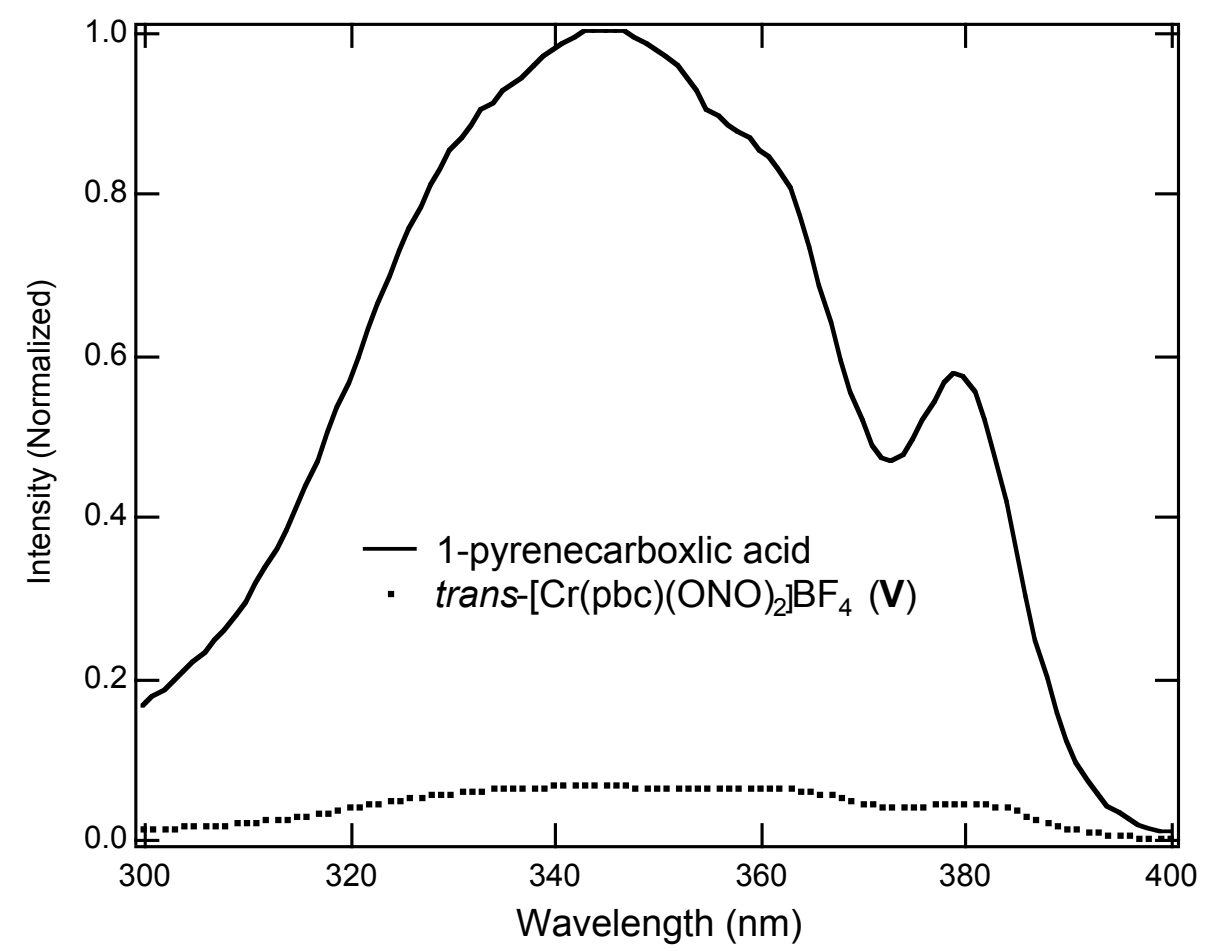


Table S1. Infrared absorption data of chromium(III) nitrito complexes in $\mathrm{KBr}$.

\begin{tabular}{|c|c|c|c|c|}
\hline Complex & $v_{\text {as }}\left(\mathrm{cm}^{-1}\right)$ & $v_{\mathrm{s}}\left(\mathrm{cm}^{-1}\right)$ & $\delta_{\text {wag }}\left(\mathrm{cm}^{-1}\right)$ & Reference \\
\hline trans $-\left[\mathrm{Cr}(\mathrm{en})_{2}(\mathrm{ONO})_{2}\right]^{+}$ & 1485 & --- & 825,835 & 15 \\
\hline trans $-\left[\mathrm{Cr}(\operatorname{cyclam})(\mathrm{ONO})_{2}\right]^{+}(\mathbf{I})$ & 1493 & 987 & 827 & 13 \\
\hline trans $-\left[\mathrm{Cr}(\text { teta })(\mathrm{ONO})_{2}\right]^{+}$ & 1492 & 986 & 825 & 13 \\
\hline trans $-\left[\mathrm{Cr}(\mathrm{mbc})(\mathrm{ONO})_{2}\right]^{+}(\mathbf{I I})$ & 1496 & 982 & 818 & This work \\
\hline trans $-\left[\mathrm{Cr}(\mathrm{mac})(\mathrm{ONO})_{2}\right]^{+}(\mathbf{I I I})$ & 1506 & 979 & 818 & This work \\
\hline trans $-\left[\mathrm{Cr}(\mathrm{hbc})(\mathrm{ONO})_{2}\right]^{+}(\mathbf{I V})$ & 1482 & 989 & 823 & This work \\
\hline trans $-\left[\mathrm{Cr}(\mathrm{pbc})(\mathrm{ONO})_{2}\right]^{+}(\mathbf{V})$ & 1493 & 985 & 820 & This work \\
\hline
\end{tabular}




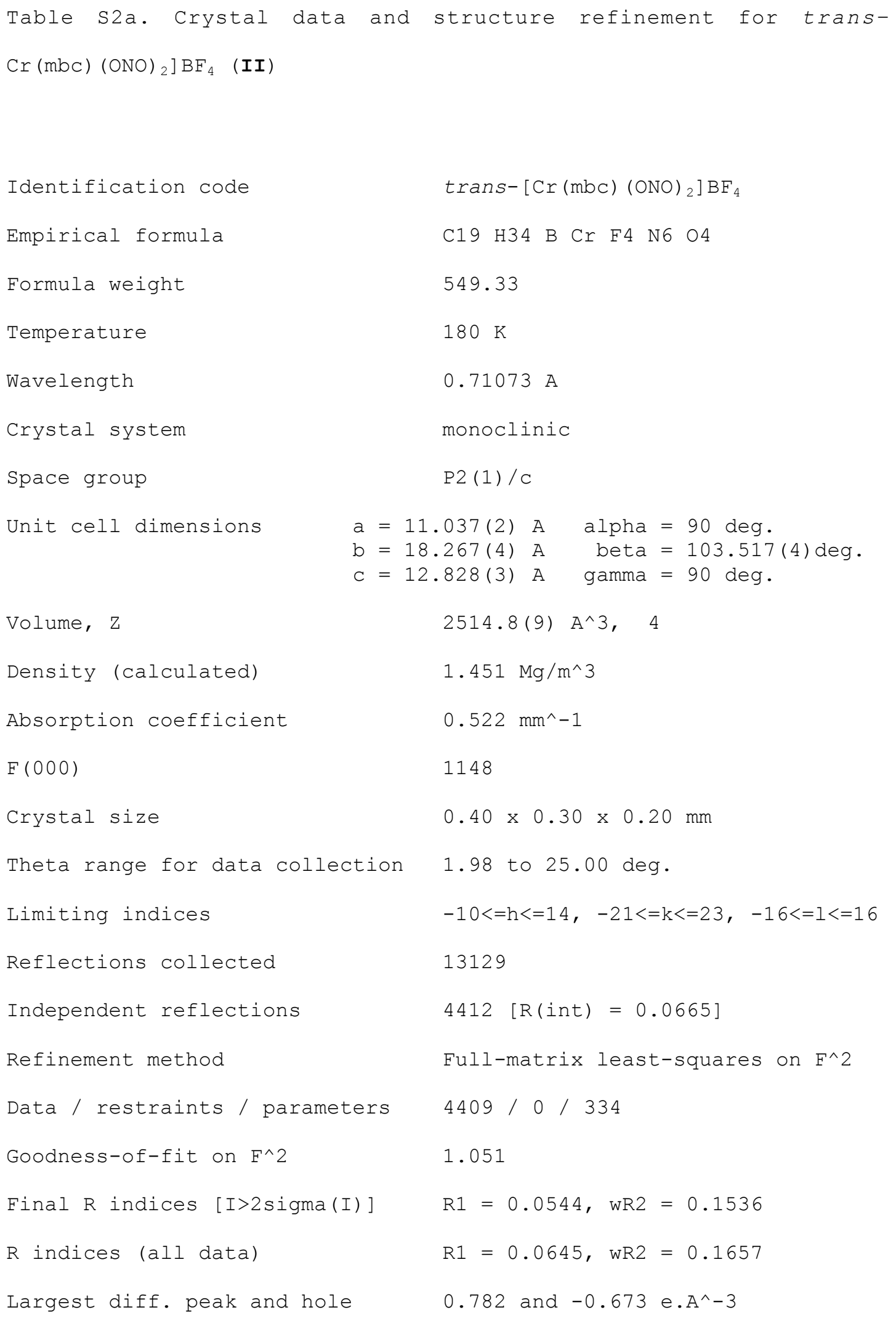


Table S2b. Atomic coordinates ( $\left.\mathrm{x} 10^{\wedge} 4\right)$ and equivalent

isotropic displacement parameters $\left(A^{\wedge} 2 \times 10^{\wedge} 3\right)$ for trans- $\mathrm{Cr}(\mathrm{mbc})(\mathrm{ONO})_{2} \mathrm{BB}_{4}$ (II).

U(eq) is defined as one third of the trace of the orthogonalized Uij tensor.

\begin{tabular}{|c|c|c|c|c|}
\hline & $\mathrm{x}$ & $\mathrm{Y}$ & $z$ & $\mathrm{U}(\mathrm{eq})$ \\
\hline $\operatorname{Cr}(1)$ & $1602(1)$ & 695 (1) & $2745(1)$ & $26(1)$ \\
\hline$O(1)$ & $1491(2)$ & $907(1)$ & $4205(2)$ & $40(1)$ \\
\hline C (1) & $-1253(3)$ & $1489(2)$ & $2198(2)$ & $33(1)$ \\
\hline $\mathrm{F}(1)$ & $7158(6)$ & $1187(3)$ & $4797(3)$ & $196(3)$ \\
\hline $\mathrm{N}(1)$ & $2477(4)$ & $921(2)$ & $4987(3)$ & $78(1)$ \\
\hline B (1) & $6449(5)$ & $1840(3)$ & $4676(3)$ & $57(1)$ \\
\hline$C(2)$ & $4441(3)$ & $-97(2)$ & $3193(3)$ & $42(1)$ \\
\hline $\mathrm{N}(2)$ & $1095(3)$ & $781(2)$ & $441(3)$ & $60(1)$ \\
\hline$O(2)$ & $2328(3)$ & $1086(2)$ & $5826(2)$ & $72(1)$ \\
\hline $\mathrm{F}(2)$ & $5361(3)$ & $1624(3)$ & $4782(2)$ & $144(2)$ \\
\hline$O(3)$ & $1674(2)$ & $426(1)$ & $1272(2)$ & $38(1)$ \\
\hline $\mathrm{N}(3)$ & $1018(2)$ & $1748(1)$ & $2314(2)$ & $29(1)$ \\
\hline$F(3)$ & $7044(4)$ & $2244(2)$ & $5484(2)$ & $135(2)$ \\
\hline $\mathrm{N}(4)$ & $3384(2)$ & $1107(1)$ & $2988(2)$ & 31 (1) \\
\hline$O(4)$ & $1216(3)$ & $562(2)$ & $-416(2)$ & $55(1)$ \\
\hline F ( 4) & $6456(2)$ & $2081(2)$ & $3676(2)$ & $69(1)$ \\
\hline $\mathrm{N}(5)$ & $-173(2)$ & $264(1)$ & $2481(2)$ & $31(1)$ \\
\hline $\mathrm{N}(6)$ & $2208(2)$ & $-356(1)$ & $3183(2)$ & $33(1)$ \\
\hline C (11) & $-3080(3)$ & $1498(2)$ & $523(2)$ & $34(1)$ \\
\hline C (12) & $-3743(3)$ & $2117(2)$ & $698(3)$ & $43(1)$ \\
\hline$C(13)$ & $-5024(3)$ & $2153(2)$ & $328(3)$ & $53(1)$ \\
\hline C (14) & $-5673(3)$ & $1575(3)$ & $-217(3)$ & $58(1)$ \\
\hline$C(15)$ & $-5035(3)$ & $967(2)$ & $-409(3)$ & $53(1)$ \\
\hline C (16) & $-3740(3)$ & $922(2)$ & $-42(3)$ & $42(1)$ \\
\hline C (17) & $-1679(3)$ & $1462(2)$ & $958(2)$ & $36(1)$ \\
\hline C (21) & $-444(3)$ & $2793(2)$ & $2306(3)$ & $44(1)$ \\
\hline C (22) & $-142(3)$ & $1996(2)$ & $2628(2)$ & $34(1)$ \\
\hline C (23) & $2132(3)$ & $2216(2)$ & $2737(3)$ & $34(1)$ \\
\hline C (24) & 3257 (3) & $1846(2)$ & $2493(3)$ & $36(1)$ \\
\hline C (25) & $4306(3)$ & $640(2)$ & $2636(3)$ & $40(1)$ \\
\hline C (31) & $-2409(3)$ & $340(2)$ & $2536(3)$ & $50(1)$ \\
\hline C (32) & $-1157(3)$ & $735(2)$ & $2748(3)$ & $37(1)$ \\
\hline$C(33)$ & $-36(3)$ & $-468(2)$ & $3017(3)$ & $40(1)$ \\
\hline C (34) & $1104(3)$ & $-838(2)$ & $2791(3)$ & $40(1)$ \\
\hline$C(35)$ & $3339(3)$ & $-615(2)$ & $2853(3)$ & $40(1)$ \\
\hline
\end{tabular}


Table S2c. Bond lengths [A] and angles [deg] for trans$\left.\mathrm{Cr}(\mathrm{mbc})(\mathrm{ONO})_{2}\right] \mathrm{BF}_{4}$ (II) .

\begin{tabular}{|c|c|}
\hline $\mathrm{Cr}(1)-\mathrm{O}(1)$ & $1.944(2)$ \\
\hline $\operatorname{Cr}(1)-O(3)$ & $1.971(2)$ \\
\hline $\mathrm{Cr}(1)-\mathrm{N}(4)$ & $2.060(2)$ \\
\hline $\mathrm{Cr}(1)-\mathrm{N}(3)$ & $2.064(3)$ \\
\hline $\operatorname{Cr}(1)-\mathrm{N}(5)$ & $2.065(2)$ \\
\hline $\operatorname{Cr}(1)-\mathrm{N}(6)$ & $2.067(3)$ \\
\hline $\mathrm{O}(1)-\mathrm{N}(1)$ & $1.296(5)$ \\
\hline$C(1)-C(22)$ & $1.533(4)$ \\
\hline$C(1)-C(32)$ & $1.539(4)$ \\
\hline$C(1)-C(17)$ & 1.551 (4) \\
\hline $\mathrm{C}(1)-\mathrm{H}(1)$ & 0.98 \\
\hline $\mathrm{F}(1)-\mathrm{B}(1)$ & $1.416(7)$ \\
\hline$N(1)-O(2)$ & $1.166(4)$ \\
\hline$B(1)-F(2)$ & $1.302(6)$ \\
\hline$B(1)-F(3)$ & $1.315(5)$ \\
\hline$B(1)-F(4)$ & $1.357(5)$ \\
\hline$C(2)-C(25)$ & $1.515(5)$ \\
\hline$C(2)-C(35)$ & $1.522(5)$ \\
\hline $\mathrm{C}(2)-\mathrm{H}(2 \mathrm{~A})$ & 0.97 \\
\hline $\mathrm{C}(2)-\mathrm{H}(2 \mathrm{~B})$ & 0.97 \\
\hline$N(2)-O(4)$ & $1.206(4)$ \\
\hline$N(2)-O(3)$ & $1.284(4)$ \\
\hline$N(3)-C(23)$ & $1.492(4)$ \\
\hline$N(3)-C(22)$ & $1.500(4)$ \\
\hline $\mathrm{N}(3)-\mathrm{H}(3)$ & $0.73(3)$ \\
\hline$N(4)-C(25)$ & $1.478(4)$ \\
\hline$N(4)-C(24)$ & $1.484(4)$ \\
\hline $\mathrm{N}(4)-\mathrm{H}(4)$ & $0.80(3)$ \\
\hline$N(5)-C(32)$ & $1.487(4)$ \\
\hline$N(5)-C(33)$ & $1.495(4)$ \\
\hline $\mathrm{N}(5)-\mathrm{H}(5)$ & $0.84(4)$ \\
\hline$N(6)-C(35)$ & $1.486(4)$ \\
\hline$N(6)-C(34)$ & $1.492(4)$ \\
\hline $\mathrm{N}(6)-\mathrm{H}(6)$ & $0.86(3)$ \\
\hline$C(11)-C(16)$ & $1.385(5)$ \\
\hline $\mathrm{C}(11)-\mathrm{C}(12)$ & $1.392(5)$ \\
\hline$C(11)-C(17)$ & $1.518(4)$ \\
\hline$C(12)-C(13)$ & $1.384(5)$ \\
\hline $\mathrm{C}(12)-\mathrm{H}(12)$ & 0.93 \\
\hline$C(13)-C(14)$ & $1.373(6)$ \\
\hline $\mathrm{C}(13)-\mathrm{H}(13)$ & 0.93 \\
\hline$C(14)-C(15)$ & $1.368(6)$ \\
\hline $\mathrm{C}(14)-\mathrm{H}(14)$ & 0.93 \\
\hline
\end{tabular}




\begin{tabular}{|c|c|}
\hline$C(15)-C(16)$ & $1.398(5)$ \\
\hline $\mathrm{C}(15)-\mathrm{H}(15)$ & 0.93 \\
\hline $\mathrm{C}(16)-\mathrm{H}(16)$ & 0.93 \\
\hline $\mathrm{C}(17)-\mathrm{H}(17 \mathrm{~A})$ & 0.97 \\
\hline $\mathrm{C}(17)-\mathrm{H}(17 \mathrm{~B})$ & 0.97 \\
\hline$C(21)-C(22)$ & $1.528(4)$ \\
\hline$C(21)-H(21 A)$ & 0.96 \\
\hline $\mathrm{C}(21)-\mathrm{H}(21 \mathrm{~B})$ & 0.96 \\
\hline $\mathrm{C}(21)-\mathrm{H}(21 \mathrm{C})$ & 0.96 \\
\hline $\mathrm{C}(22)-\mathrm{H}(22)$ & 0.98 \\
\hline$C(23)-C(24)$ & $1.509(4)$ \\
\hline$C(23)-H(23 A)$ & 0.97 \\
\hline$C(23)-H(23 B)$ & 0.97 \\
\hline $\mathrm{C}(24)-\mathrm{H}(24 \mathrm{~A})$ & 0.97 \\
\hline$C(24)-H(24 B)$ & 0.97 \\
\hline$C(25)-H(25 A)$ & 0.97 \\
\hline$C(25)-H(25 B)$ & 0.97 \\
\hline$C(31)-C(32)$ & $1.525(5)$ \\
\hline $\mathrm{C}(31)-\mathrm{H}(31 \mathrm{~A})$ & 0.96 \\
\hline $\mathrm{C}(31)-\mathrm{H}(31 \mathrm{~B})$ & 0.96 \\
\hline $\mathrm{C}(31)-\mathrm{H}(31 \mathrm{C})$ & 0.96 \\
\hline $\mathrm{C}(32)-\mathrm{H}(32)$ & 0.98 \\
\hline$C(33)-C(34)$ & $1.514(5)$ \\
\hline $\mathrm{C}(33)-\mathrm{H}(33 \mathrm{~A})$ & 0.97 \\
\hline$C(33)-H(33 B)$ & 0.97 \\
\hline $\mathrm{C}(34)-\mathrm{H}(34 \mathrm{~A})$ & 0.97 \\
\hline$C(34)-H(34 B)$ & 0.97 \\
\hline $\mathrm{C}(35)-\mathrm{H}(35 \mathrm{~A})$ & 0.97 \\
\hline $\mathrm{C}(35)-\mathrm{H}(35 \mathrm{~B})$ & 0.97 \\
\hline $\mathrm{O}(1)-\mathrm{Cr}(1)-\mathrm{O}(3)$ & $176.80(10)$ \\
\hline $\mathrm{O}(1)-\mathrm{Cr}(1)-\mathrm{N}(4)$ & $93.12(10)$ \\
\hline $\mathrm{O}(3)-\mathrm{Cr}(1)-\mathrm{N}(4)$ & $89.17(10)$ \\
\hline $\mathrm{O}(1)-\mathrm{Cr}(1)-\mathrm{N}(3)$ & $89.22(10)$ \\
\hline $\mathrm{O}(3)-\mathrm{Cr}(1)-\mathrm{N}(3)$ & $93.18(10)$ \\
\hline$N(4)-\operatorname{Cr}(1)-N(3)$ & $85.80(10)$ \\
\hline $\mathrm{O}(1)-\mathrm{Cr}(1)-\mathrm{N}(5)$ & $87.87(10)$ \\
\hline $\mathrm{O}(3)-\mathrm{Cr}(1)-\mathrm{N}(5)$ & $89.81(10)$ \\
\hline$N(4)-\operatorname{Cr}(1)-N(5)$ & $178.76(10)$ \\
\hline$N(3)-\operatorname{Cr}(1)-N(5)$ & $94.96(10)$ \\
\hline $\mathrm{O}(1)-\mathrm{Cr}(1)-\mathrm{N}(6)$ & $90.73(11)$ \\
\hline $\mathrm{O}(3)-\mathrm{Cr}(1)-\mathrm{N}(6)$ & $86.90(10)$ \\
\hline$N(4)-\operatorname{Cr}(1)-N(6)$ & $93.55(10)$ \\
\hline$N(3)-\operatorname{Cr}(1)-N(6)$ & $179.34(10)$ \\
\hline$N(5)-\operatorname{Cr}(1)-N(6)$ & $85.69(10)$ \\
\hline $\mathrm{N}(1)-\mathrm{O}(1)-\mathrm{Cr}(1)$ & $121.2(2)$ \\
\hline$C(22)-C(1)-C(32)$ & $114.4(2)$ \\
\hline$C(22)-C(1)-C(17)$ & $114.4(3)$ \\
\hline$C(32)-C(1)-C(17)$ & $114.4(3)$ \\
\hline $\mathrm{C}(22)-\mathrm{C}(1)-\mathrm{H}(1)$ & $103.9(2)$ \\
\hline $\mathrm{C}(32)-\mathrm{C}(1)-\mathrm{H}(1)$ & $103.9(2)$ \\
\hline $\mathrm{C}(17)-\mathrm{C}(1)-\mathrm{H}(1)$ & $103.9(2)$ \\
\hline $\mathrm{O}(2)-\mathrm{N}(1)-\mathrm{O}(1)$ & $116.5(4)$ \\
\hline$F(2)-B(1)-F(3)$ & $113.0(4)$ \\
\hline
\end{tabular}




\begin{tabular}{|c|c|}
\hline$F(2)-B(1)-F(4)$ & $114.0(4)$ \\
\hline$F(3)-B(1)-F(4)$ & $116.8(4)$ \\
\hline$F(2)-B(1)-F(1)$ & $103.4(5)$ \\
\hline$F(3)-B(1)-F(1)$ & $103.0(5)$ \\
\hline$F(4)-B(1)-F(1)$ & $104.6(4)$ \\
\hline$C(25)-C(2)-C(35)$ & $115.7(3)$ \\
\hline $\mathrm{C}(25)-\mathrm{C}(2)-\mathrm{H}(2 \mathrm{~A})$ & $108.4(2)$ \\
\hline $\mathrm{C}(35)-\mathrm{C}(2)-\mathrm{H}(2 \mathrm{~A})$ & $108.4(2)$ \\
\hline$C(25)-C(2)-H(2 B)$ & $108.4(2)$ \\
\hline$C(35)-C(2)-H(2 B)$ & $108.4(2)$ \\
\hline $\mathrm{H}(2 \mathrm{~A})-\mathrm{C}(2)-\mathrm{H}(2 \mathrm{~B})$ & 107.4 \\
\hline $\mathrm{O}(4)-\mathrm{N}(2)-\mathrm{O}(3)$ & $116.8(3)$ \\
\hline $\mathrm{N}(2)-\mathrm{O}(3)-\mathrm{Cr}(1)$ & $123.2(2)$ \\
\hline$C(23)-N(3)-C(22)$ & $114.3(2)$ \\
\hline $\mathrm{C}(23)-\mathrm{N}(3)-\mathrm{Cr}(1)$ & $104.9(2)$ \\
\hline $\mathrm{C}(22)-\mathrm{N}(3)-\mathrm{Cr}(1)$ & $116.3(2)$ \\
\hline $\mathrm{C}(23)-\mathrm{N}(3)-\mathrm{H}(3)$ & $106(2)$ \\
\hline $\mathrm{C}(22)-\mathrm{N}(3)-\mathrm{H}(3)$ & $107(2)$ \\
\hline $\operatorname{Cr}(1)-\mathrm{N}(3)-\mathrm{H}(3)$ & $107(2)$ \\
\hline$C(25)-N(4)-C(24)$ & $113.4(2)$ \\
\hline $\mathrm{C}(25)-\mathrm{N}(4)-\mathrm{Cr}(1)$ & $116.3(2)$ \\
\hline $\mathrm{C}(24)-\mathrm{N}(4)-\mathrm{Cr}(1)$ & $106.1(2)$ \\
\hline $\mathrm{C}(25)-\mathrm{N}(4)-\mathrm{H}(4)$ & $109(2)$ \\
\hline $\mathrm{C}(24)-\mathrm{N}(4)-\mathrm{H}(4)$ & $109(2)$ \\
\hline $\operatorname{Cr}(1)-\mathrm{N}(4)-\mathrm{H}(4)$ & $103(2)$ \\
\hline$C(32)-N(5)-C(33)$ & $114.4(2)$ \\
\hline $\mathrm{C}(32)-\mathrm{N}(5)-\mathrm{Cr}(1)$ & $117.2(2)$ \\
\hline $\mathrm{C}(33)-\mathrm{N}(5)-\mathrm{Cr}(1)$ & $106.0(2)$ \\
\hline $\mathrm{C}(32)-\mathrm{N}(5)-\mathrm{H}(5)$ & $104(2)$ \\
\hline $\mathrm{C}(33)-\mathrm{N}(5)-\mathrm{H}(5)$ & $109(2)$ \\
\hline $\operatorname{Cr}(1)-\mathrm{N}(5)-\mathrm{H}(5)$ & $105(2)$ \\
\hline$C(35)-N(6)-C(34)$ & $113.0(3)$ \\
\hline $\mathrm{C}(35)-\mathrm{N}(6)-\mathrm{Cr}(1)$ & $117.3(2)$ \\
\hline $\mathrm{C}(34)-\mathrm{N}(6)-\mathrm{Cr}(1)$ & $105.6(2)$ \\
\hline $\mathrm{C}(35)-\mathrm{N}(6)-\mathrm{H}(6)$ & $107(2)$ \\
\hline $\mathrm{C}(34)-\mathrm{N}(6)-\mathrm{H}(6)$ & $107(2)$ \\
\hline $\mathrm{Cr}(1)-\mathrm{N}(6)-\mathrm{H}(6)$ & $106(2)$ \\
\hline$C(16)-C(11)-C(12)$ & $118.0(3)$ \\
\hline$C(16)-C(11)-C(17)$ & $121.7(3)$ \\
\hline$C(12)-C(11)-C(17)$ & $120.3(3)$ \\
\hline$C(13)-C(12)-C(11)$ & $121.2(4)$ \\
\hline $\mathrm{C}(13)-\mathrm{C}(12)-\mathrm{H}(12)$ & $119.4(2)$ \\
\hline $\mathrm{C}(11)-\mathrm{C}(12)-\mathrm{H}(12)$ & $119.4(2)$ \\
\hline$C(14)-C(13)-C(12)$ & $120.4(4)$ \\
\hline $\mathrm{C}(14)-\mathrm{C}(13)-\mathrm{H}(13)$ & $119.8(2)$ \\
\hline $\mathrm{C}(12)-\mathrm{C}(13)-\mathrm{H}(13)$ & $119.8(2)$ \\
\hline$C(15)-C(14)-C(13)$ & $119.2(3)$ \\
\hline $\mathrm{C}(15)-\mathrm{C}(14)-\mathrm{H}(14)$ & $120.4(2)$ \\
\hline $\mathrm{C}(13)-\mathrm{C}(14)-\mathrm{H}(14)$ & $120.4(2)$ \\
\hline$C(14)-C(15)-C(16)$ & $121.1(4)$ \\
\hline $\mathrm{C}(14)-\mathrm{C}(15)-\mathrm{H}(15)$ & $119.5(2)$ \\
\hline $\mathrm{C}(16)-\mathrm{C}(15)-\mathrm{H}(15)$ & $119.5(2)$ \\
\hline$C(11)-C(16)-C(15)$ & $120.2(3)$ \\
\hline $\mathrm{C}(11)-\mathrm{C}(16)-\mathrm{H}(16)$ & $119.9(2)$ \\
\hline
\end{tabular}




\begin{tabular}{|c|c|}
\hline $\mathrm{C}(15)-\mathrm{C}(16)-\mathrm{H}(16)$ & $119.9(2)$ \\
\hline$C(11)-C(17)-C(1)$ & $114.5(2)$ \\
\hline $\mathrm{C}(11)-\mathrm{C}(17)-\mathrm{H}(17 \mathrm{~A})$ & $108.6(2)$ \\
\hline $\mathrm{C}(1)-\mathrm{C}(17)-\mathrm{H}(17 \mathrm{~A})$ & $108.6(2)$ \\
\hline $\mathrm{C}(11)-\mathrm{C}(17)-\mathrm{H}(17 \mathrm{~B})$ & $108.6(2)$ \\
\hline $\mathrm{C}(1)-\mathrm{C}(17)-\mathrm{H}(17 \mathrm{~B})$ & $108.6(2)$ \\
\hline $\mathrm{H}(17 \mathrm{~A})-\mathrm{C}(17)-\mathrm{H}(17 \mathrm{~B})$ & 107.6 \\
\hline $\mathrm{C}(22)-\mathrm{C}(21)-\mathrm{H}(21 \mathrm{~A})$ & $109.5(2)$ \\
\hline$C(22)-C(21)-H(21 B)$ & $109.5(2)$ \\
\hline $\mathrm{H}(21 \mathrm{~A})-\mathrm{C}(21)-\mathrm{H}(21 \mathrm{~B})$ & 109.5 \\
\hline $\mathrm{C}(22)-\mathrm{C}(21)-\mathrm{H}(21 \mathrm{C})$ & $109.5(2)$ \\
\hline $\mathrm{H}(21 \mathrm{~A})-\mathrm{C}(21)-\mathrm{H}(21 \mathrm{C})$ & 109.5 \\
\hline $\mathrm{H}(21 \mathrm{~B})-\mathrm{C}(21)-\mathrm{H}(21 \mathrm{C})$ & 109.5 \\
\hline$N(3)-C(22)-C(21)$ & $111.2(3)$ \\
\hline$N(3)-C(22)-C(1)$ & $112.5(2)$ \\
\hline$C(21)-C(22)-C(1)$ & $112.0(3)$ \\
\hline $\mathrm{N}(3)-\mathrm{C}(22)-\mathrm{H}(22)$ & $107.0(2)$ \\
\hline $\mathrm{C}(21)-\mathrm{C}(22)-\mathrm{H}(22)$ & $107.0(2)$ \\
\hline $\mathrm{C}(1)-\mathrm{C}(22)-\mathrm{H}(22)$ & $107.0(2)$ \\
\hline$N(3)-C(23)-C(24)$ & $108.4(2)$ \\
\hline $\mathrm{N}(3)-\mathrm{C}(23)-\mathrm{H}(23 \mathrm{~A})$ & $110.0(2)$ \\
\hline$C(24)-C(23)-H(23 A)$ & $110.0(2)$ \\
\hline$N(3)-C(23)-H(23 B)$ & $110.0(2)$ \\
\hline $\mathrm{C}(24)-\mathrm{C}(23)-\mathrm{H}(23 \mathrm{~B})$ & $110.0(2)$ \\
\hline $\mathrm{H}(23 \mathrm{~A})-\mathrm{C}(23)-\mathrm{H}(23 \mathrm{~B})$ & 108.4 \\
\hline$N(4)-C(24)-C(23)$ & $108 \cdot 6(2)$ \\
\hline $\mathrm{N}(4)-\mathrm{C}(24)-\mathrm{H}(24 \mathrm{~A})$ & $110.0(2)$ \\
\hline$C(23)-C(24)-H(24 A)$ & $110.0(2)$ \\
\hline$N(4)-C(24)-H(24 B)$ & $110.0(2)$ \\
\hline $\mathrm{C}(23)-\mathrm{C}(24)-\mathrm{H}(24 \mathrm{~B})$ & $110.0(2)$ \\
\hline $\mathrm{H}(24 \mathrm{~A})-\mathrm{C}(24)-\mathrm{H}(24 \mathrm{~B})$ & 108.4 \\
\hline$N(4)-C(25)-C(2)$ & $111.6(3)$ \\
\hline$N(4)-C(25)-H(25 A)$ & $109.3(2)$ \\
\hline$C(2)-C(25)-H(25 A)$ & $109.3(2)$ \\
\hline$N(4)-C(25)-H(25 B)$ & $109.3(2)$ \\
\hline $\mathrm{C}(2)-\mathrm{C}(25)-\mathrm{H}(25 \mathrm{~B})$ & $109.3(2)$ \\
\hline $\mathrm{H}(25 \mathrm{~A})-\mathrm{C}(25)-\mathrm{H}(25 \mathrm{~B})$ & 108.0 \\
\hline $\mathrm{C}(32)-\mathrm{C}(31)-\mathrm{H}(31 \mathrm{~A})$ & $109.5(2)$ \\
\hline $\mathrm{C}(32)-\mathrm{C}(31)-\mathrm{H}(31 \mathrm{~B})$ & $109.5(2)$ \\
\hline $\mathrm{H}(31 \mathrm{~A})-\mathrm{C}(31)-\mathrm{H}(31 \mathrm{~B})$ & 109.5 \\
\hline $\mathrm{C}(32)-\mathrm{C}(31)-\mathrm{H}(31 \mathrm{C})$ & $109.5(2)$ \\
\hline $\mathrm{H}(31 \mathrm{~A})-\mathrm{C}(31)-\mathrm{H}(31 \mathrm{C})$ & 109.5 \\
\hline $\mathrm{H}(31 \mathrm{~B})-\mathrm{C}(31)-\mathrm{H}(31 \mathrm{C})$ & 109.5 \\
\hline$N(5)-C(32)-C(31)$ & $111.4(3)$ \\
\hline $\mathrm{N}(5)-\mathrm{C}(32)-\mathrm{C}(1)$ & $112.8(2)$ \\
\hline$C(31)-C(32)-C(1)$ & $112.3(3)$ \\
\hline $\mathrm{N}(5)-\mathrm{C}(32)-\mathrm{H}(32)$ & $106.6(2)$ \\
\hline $\mathrm{C}(31)-\mathrm{C}(32)-\mathrm{H}(32)$ & $106.6(2)$ \\
\hline $\mathrm{C}(1)-\mathrm{C}(32)-\mathrm{H}(32)$ & $106.6(2)$ \\
\hline$N(5)-C(33)-C(34)$ & $108.1(2)$ \\
\hline$N(5)-C(33)-H(33 A)$ & $110.1(2)$ \\
\hline $\mathrm{C}(34)-\mathrm{C}(33)-\mathrm{H}(33 \mathrm{~A})$ & $110.1(2)$ \\
\hline$N(5)-C(33)-H(33 B)$ & $110.1(2)$ \\
\hline $\mathrm{C}(34)-\mathrm{C}(33)-\mathrm{H}(33 \mathrm{~B})$ & $110.1(2)$ \\
\hline
\end{tabular}




$\begin{array}{ll}\mathrm{H}(33 \mathrm{~A})-\mathrm{C}(33)-\mathrm{H}(33 \mathrm{~B}) & 108.4 \\ \mathrm{~N}(6)-\mathrm{C}(34)-\mathrm{C}(33) & 108.8(3) \\ \mathrm{N}(6)-\mathrm{C}(34)-\mathrm{H}(34 \mathrm{~A}) & 109.9(2) \\ \mathrm{C}(33)-\mathrm{C}(34)-\mathrm{H}(34 \mathrm{~A}) & 109.9(2) \\ \mathrm{N}(6)-\mathrm{C}(34)-\mathrm{H}(34 \mathrm{~B}) & 109.9(2) \\ \mathrm{C}(33)-\mathrm{C}(34)-\mathrm{H}(34 \mathrm{~B}) & 109.9(2) \\ \mathrm{H}(34 \mathrm{~A})-\mathrm{C}(34)-\mathrm{H}(34 \mathrm{~B}) & 108.3 \\ \mathrm{~N}(6)-\mathrm{C}(35)-\mathrm{C}(2) & 112.9(3) \\ \mathrm{N}(6)-\mathrm{C}(35)-\mathrm{H}(35 \mathrm{~A}) & 109.0(2) \\ \mathrm{C}(2)-\mathrm{C}(35)-\mathrm{H}(35 \mathrm{~A}) & 109.0(2) \\ \mathrm{N}(6)-\mathrm{C}(35)-\mathrm{H}(35 \mathrm{~B}) & 109.0(2) \\ \mathrm{C}(2)-\mathrm{C}(35)-\mathrm{H}(35 \mathrm{~B}) & 109.0(2) \\ \mathrm{H}(35 \mathrm{~A})-\mathrm{C}(35)-\mathrm{H}(35 \mathrm{~B}) & 107.8\end{array}$

Symmetry transformations used to generate equivalent atoms: 
Table S2d. Anisotropic displacement parameters $\left(\mathrm{A}^{\wedge} 2 \times 10^{\wedge} 3\right)$ for trans$\left.\mathrm{Cr}(\mathrm{mbc})(\mathrm{ONO})_{2}\right]_{\mathrm{BF}_{4}}(\mathbf{I I})$. The anisotropic displacement factor exponent takes the form: $-2 \mathrm{pi}^{\wedge} 2$ [ $\mathrm{h}^{\wedge} 2 \mathrm{a}^{* \wedge} 2 \mathrm{U} 11+\ldots+2 \mathrm{~h} \mathrm{k} \mathrm{a} \mathrm{a}^{*} \mathrm{U} 12$ ]

\begin{tabular}{|c|c|c|c|c|c|c|}
\hline & $\mathrm{U} 11$ & $\mathrm{U} 22$ & U33 & U23 & U13 & $\mathrm{U} 12$ \\
\hline $\operatorname{Cr}(1)$ & $17(1)$ & $28(1)$ & $33(1)$ & $-1(1)$ & $7(1)$ & $0(1)$ \\
\hline$O(1)$ & $34(1)$ & $56(1)$ & $29(1)$ & $-4(1)$ & $5(1)$ & $-1(1)$ \\
\hline$C(1)$ & $23(2)$ & $41(2)$ & $37(2)$ & $-1(1)$ & $12(1)$ & $8(1)$ \\
\hline F (1) & $319(8)$ & 195 ( 5) & $79(3)$ & $47(3)$ & $58(3)$ & $129(5)$ \\
\hline $\mathrm{N}(1)$ & $100(3)$ & $98(3)$ & $31(2)$ & $-7(2)$ & $8(2)$ & $-25(2)$ \\
\hline B (1) & $54(3)$ & $73(3)$ & $38(2)$ & $1(2)$ & $-1(2)$ & $-25(2)$ \\
\hline C (2) & $24(2)$ & $46(2)$ & $56(2)$ & $0(2)$ & $9(1)$ & $9(1)$ \\
\hline $\mathrm{N}(2)$ & $56(2)$ & $81(2)$ & $42(2)$ & $-5(2)$ & $11(2)$ & $19(2)$ \\
\hline$O(2)$ & $93(2)$ & $71(2)$ & $53(2)$ & $6(2)$ & $17(2)$ & $-15(2)$ \\
\hline$F(2)$ & $90(2)$ & $286(6)$ & $53(2)$ & $-12(2)$ & $9(2)$ & $-110(3)$ \\
\hline O (3) & $36(1)$ & $44(1)$ & $33(1)$ & $0(1)$ & $7(1)$ & $6(1)$ \\
\hline $\mathrm{N}(3)$ & $25(1)$ & $33(1)$ & $29(1)$ & $-2(1)$ & $7(1)$ & $0(1)$ \\
\hline F (3) & $155(3)$ & $188(4)$ & $64(2)$ & $-43(2)$ & $30(2)$ & $-121(3)$ \\
\hline $\mathrm{N}(4)$ & $21(1)$ & $39(1)$ & $31(1)$ & $0(1)$ & $5(1)$ & $-1(1)$ \\
\hline$O(4)$ & $55(2)$ & $71(2)$ & $38(1)$ & $-11(1)$ & $10(1)$ & $-7(1)$ \\
\hline F (4) & $74(2)$ & $91(2)$ & $44(1)$ & $6(1)$ & $16(1)$ & $-11(1)$ \\
\hline$N(5)$ & $20(1)$ & $35(1)$ & $37(2)$ & $2(1)$ & $8(1)$ & $-1(1)$ \\
\hline $\mathrm{N}(6)$ & $25(1)$ & $34(1)$ & $38(2)$ & $0(1)$ & $6(1)$ & $3(1)$ \\
\hline$C(11)$ & $27(2)$ & $42(2)$ & $32(2)$ & $6(1)$ & $8(1)$ & $2(1)$ \\
\hline$C(12)$ & $33(2)$ & $48(2)$ & $48(2)$ & $5(2)$ & $8(1)$ & $7(2)$ \\
\hline$C(13)$ & $37(2)$ & $75(3)$ & $48(2)$ & $13(2)$ & $14(2)$ & $22(2)$ \\
\hline$C(14)$ & $26(2)$ & $97(3)$ & $48(2)$ & $22(2)$ & $6(2)$ & $0(2)$ \\
\hline$C(15)$ & $40(2)$ & $70(3)$ & $43(2)$ & $9(2)$ & $-3(2)$ & $-16(2)$ \\
\hline$C(16)$ & $41(2)$ & $46(2)$ & $37(2)$ & $2(1)$ & $4(1)$ & $1(2)$ \\
\hline$C(17)$ & $26(2)$ & $44(2)$ & $40(2)$ & $1(1)$ & $10(1)$ & $7(1)$ \\
\hline C (21) & $42(2)$ & $39(2)$ & $51(2)$ & $-6(2)$ & $11(2)$ & $10(1)$ \\
\hline C (22) & $27(2)$ & $38(2)$ & $37(2)$ & $-3(1)$ & $11(1)$ & $6(1)$ \\
\hline$C(23)$ & $30(2)$ & $28(2)$ & $42(2)$ & $0(1)$ & $8(1)$ & $-3(1)$ \\
\hline C (24) & $26(2)$ & $36(2)$ & $47(2)$ & $6(1)$ & $11(1)$ & $-5(1)$ \\
\hline$C(25)$ & $22(2)$ & $47(2)$ & $51(2)$ & $-3(2)$ & $13(1)$ & $-1(1)$ \\
\hline C (31) & $25(2)$ & $61(2)$ & $66(2)$ & $17(2)$ & $17(2)$ & $0(2)$ \\
\hline C (32) & $24(2)$ & $45(2)$ & $44(2)$ & $5(1)$ & $14(1)$ & $2(1)$ \\
\hline$C(33)$ & $30(2)$ & $36(2)$ & $54(2)$ & $7(1)$ & $13(1)$ & $-6(1)$ \\
\hline$C(34)$ & $34(2)$ & $28(2)$ & $57(2)$ & $2(1)$ & $9(2)$ & $-3(1)$ \\
\hline C (35) & $30(2)$ & $38(2)$ & $53(2)$ & $2(1)$ & $13(2)$ & $10(1)$ \\
\hline
\end{tabular}


Table s2e. Hydrogen coordinates ( $x$ 10^4) and isotropic displacement parameters $\left(A^{\wedge} 2 \times 10^{\wedge} 3\right)$ for trans$\mathrm{Cr}(\mathrm{mbc})(\mathrm{ONO})_{2} \mathrm{BF}_{4}$ (II) .

.

\begin{tabular}{|c|c|c|c|c|}
\hline & $\mathrm{x}$ & $\mathrm{y}$ & z & $\mathrm{U}(\mathrm{eq})$ \\
\hline H (1) & $-1948(3)$ & $1728(2)$ & $2419(2)$ & 39 \\
\hline $\mathrm{H}(2 \mathrm{~A})$ & $4583(3)$ & $-15(2)$ & $3959(3)$ & 50 \\
\hline $\mathrm{H}(2 \mathrm{~B})$ & 5177 (3) & $-337(2)$ & $3064(3)$ & 50 \\
\hline $\mathrm{H}(12)$ & $-3318(3)$ & $2512(2)$ & $1070(3)$ & 52 \\
\hline H (13) & $-5448(3)$ & $2573(2)$ & $450(3)$ & 63 \\
\hline H (14) & $-6536(3)$ & $1596(3)$ & $-452(3)$ & 69 \\
\hline H (15) & $-5469(3)$ & $578(2)$ & $-791(3)$ & 64 \\
\hline $\mathrm{H}(16)$ & $-3322(3)$ & $504(2)$ & $-178(3)$ & 51 \\
\hline $\mathrm{H}(17 \mathrm{~A})$ & $-1299(3)$ & $1867(2)$ & $664(2)$ & 44 \\
\hline $\mathrm{H}(17 \mathrm{~B})$ & $-1373(3)$ & $1012(2)$ & $708(2)$ & 44 \\
\hline $\mathrm{H}(21 \mathrm{~A})$ & $173(14)$ & $3107(2)$ & $2736(14)$ & 66 \\
\hline$H(21 B)$ & $-1250(11)$ & $2916(4)$ & $2417(19)$ & 66 \\
\hline $\mathrm{H}(21 \mathrm{C})$ & $-444(24)$ & $2857(4)$ & $1563(6)$ & 66 \\
\hline $\mathrm{H}(22)$ & $29(3)$ & $1975(2)$ & $3412(2)$ & 40 \\
\hline$H(23 A)$ & 2251 (3) & $2279(2)$ & 3505 (3) & 40 \\
\hline$H(23 B)$ & $2016(3)$ & $2695(2)$ & $2402(3)$ & 40 \\
\hline$H(24 A)$ & $3156(3)$ & $1804(2)$ & $1723(3)$ & 43 \\
\hline$H(24 B)$ & $3999(3)$ & $2133(2)$ & $2780(3)$ & 43 \\
\hline $\mathrm{H}(25 \mathrm{~A})$ & 5107 (3) & $886(2)$ & $2789(3)$ & 48 \\
\hline$H(25 B)$ & $4047(3)$ & $567(2)$ & $1867(3)$ & 48 \\
\hline$H(31 A)$ & $-3034(6)$ & $667(5)$ & $2675(21)$ & 74 \\
\hline $\mathrm{H}(31 \mathrm{~B})$ & $-2344(8)$ & $-78(9)$ & $2999(16)$ & 74 \\
\hline $\mathrm{H}(31 \mathrm{C})$ & $-2635(13)$ & $184(13)$ & $1802(7)$ & 74 \\
\hline $\mathrm{H}(32)$ & $-910(3)$ & $826(2)$ & $3521(3)$ & 44 \\
\hline $\mathrm{H}(33 \mathrm{~A})$ & $-771(3)$ & $-764(2)$ & $2741(3)$ & 48 \\
\hline$H(33 B)$ & $61(3)$ & $-410(2)$ & $3784(3)$ & 48 \\
\hline$H(34 A)$ & $1236(3)$ & $-1307(2)$ & $3154(3)$ & 48 \\
\hline $\mathrm{H}(34 \mathrm{~B})$ & $984(3)$ & $-922(2)$ & $2027(3)$ & 48 \\
\hline$H(35 A)$ & $3147(3)$ & $-669(2)$ & $2079(3)$ & 48 \\
\hline $\mathrm{H}(35 \mathrm{~B})$ & $3570(3)$ & $-1093(2)$ & $3166(3)$ & 48 \\
\hline $\mathrm{H}(3)$ & $900(28)$ & $1770(16)$ & $1730(26)$ & $19(8)$ \\
\hline H (4) & $3586(28)$ & $1151(16)$ & $3628(26)$ & $22(8)$ \\
\hline H (5) & $-399(31)$ & $205(18)$ & $1818(29)$ & $34(9)$ \\
\hline $\mathrm{H}(6)$ & $2377(29)$ & $-366(17)$ & $3868(28)$ & $28(8)$ \\
\hline
\end{tabular}




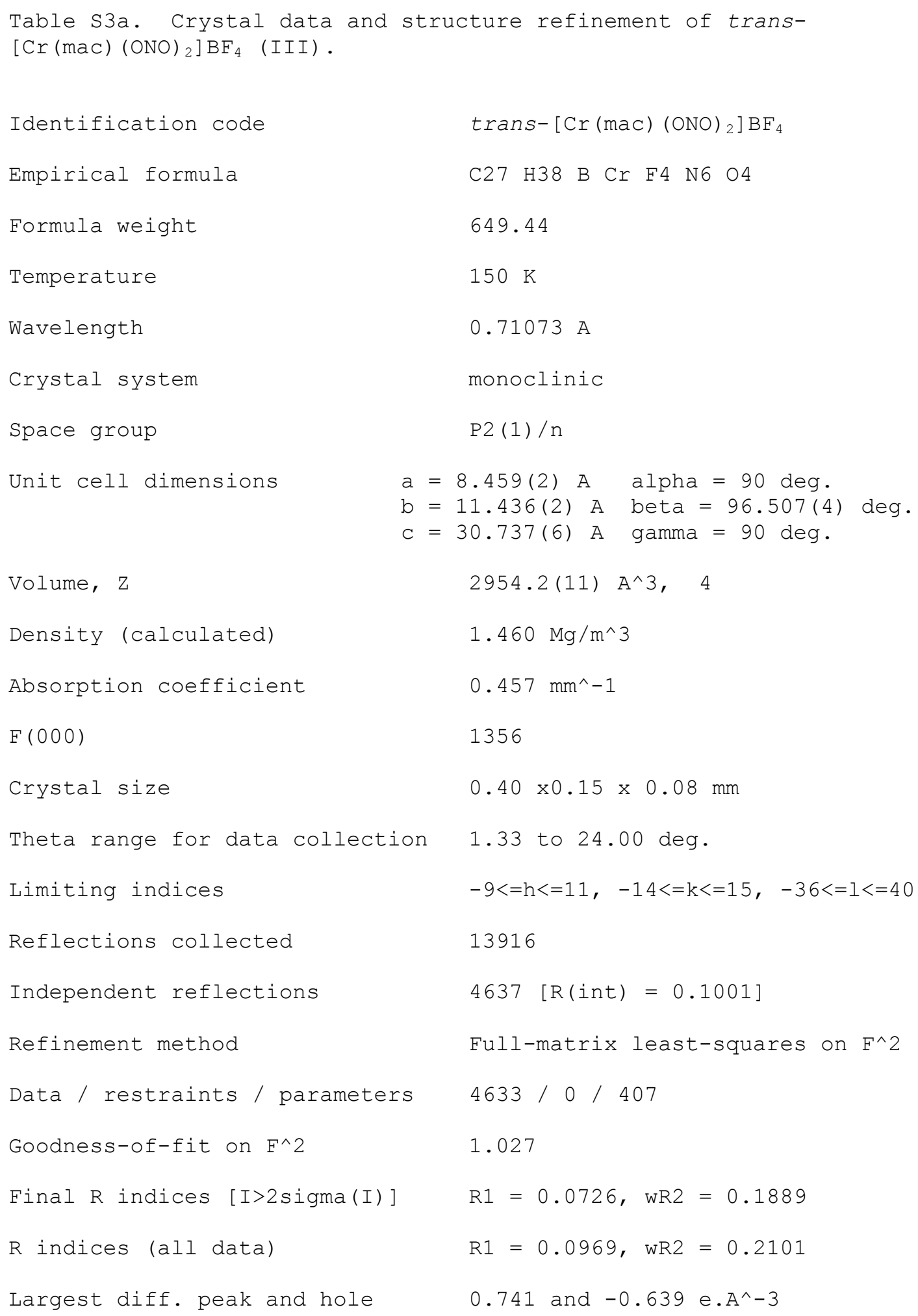


Table S3b. Atomic coordinates ( $x$ 10^4) and equivalent

isotropic displacement parameters $\left(A^{\wedge} 2 \times 10^{\wedge} 3\right)$ of trans-[Cr (mac) (ONO) $\left.{ }_{2}\right] \mathrm{BF}_{4}$ (III).

$\mathrm{U}(\mathrm{eq})$ is defined as one third of the trace of the orthogonalized Uij tensor.

\begin{tabular}{|c|c|c|c|c|}
\hline & $\mathrm{x}$ & $\mathrm{y}$ & z & $\mathrm{U}(\mathrm{eq})$ \\
\hline $\operatorname{Cr}(1)$ & $4692(1)$ & $1190(1)$ & $1788(1)$ & $37(1)$ \\
\hline$O(1)$ & $2611(5)$ & $449(4)$ & $1688(2)$ & $74(1)$ \\
\hline B (1) & $4227(7)$ & $-3868(5)$ & $1957(2)$ & $41(1)$ \\
\hline$F(1)$ & $5083(5)$ & $-2902(3)$ & $1931(2)$ & $109(2)$ \\
\hline $\mathrm{N}(1)$ & $2103(9)$ & $-430(7)$ & $1766(3)$ & $123(3)$ \\
\hline$F(2)$ & $3952(5)$ & $-4119(3)$ & $2378(1)$ & $88(1)$ \\
\hline $\mathrm{N}(2)$ & $7621(6)$ & $2149(4)$ & $1605(2)$ & $67(1)$ \\
\hline$O(2)$ & $752(6)$ & $-733(5)$ & $1617(2)$ & $104(2)$ \\
\hline$O(3)$ & $6716(4)$ & $2048(3)$ & $1893(1)$ & $45(1)$ \\
\hline$N(3)$ & $3542(5)$ & $2749(4)$ & $1613(1)$ & $34(1)$ \\
\hline$F(3)$ & $2732(5)$ & $-3734(4)$ & $1735(2)$ & $104(2)$ \\
\hline$F(4)$ & $4971(3)$ & $-4814(2)$ & $1788(1)$ & $56(1)$ \\
\hline$N(4)$ & $4320(5)$ & $1602(4)$ & $2420(1)$ & $45(1)$ \\
\hline$O(4)$ & $8848(5)$ & $2660(4)$ & $1695(1)$ & $72(1)$ \\
\hline$N(5)$ & $5017(5)$ & $739(3)$ & $1154(2)$ & $45(1)$ \\
\hline $\mathrm{N}(6)$ & $5837(7)$ & $-359(4)$ & $1968(2)$ & $57(2)$ \\
\hline C (11) & $3355(5)$ & $2366(4)$ & $805(2)$ & $38(1)$ \\
\hline C (12) & $6073(6)$ & $57(5)$ & $2774(2)$ & $60(2)$ \\
\hline$C(13)$ & $5198(5)$ & $3167(4)$ & $245(2)$ & $40(1)$ \\
\hline C (14) & $5992(7)$ & $3107(4)$ & $-625(2)$ & $51(1)$ \\
\hline C (15) & $4740(6)$ & $3200(4)$ & $710(2)$ & $42(1)$ \\
\hline$C(21)$ & $1600(6)$ & $3912(4)$ & $1114(2)$ & $45(1)$ \\
\hline C (22) & $2495(5)$ & 2755 (4) & $1196(1)$ & $36(1)$ \\
\hline C (23) & $2733(5)$ & $3070(4)$ & $2003(1)$ & $38(1)$ \\
\hline C (24) & $3835(6)$ & $2837(4)$ & $2414(2)$ & $43(1)$ \\
\hline C (25) & $5629(6)$ & $1323(5)$ & $2770(2)$ & $53(1)$ \\
\hline C (31) & $3972(7)$ & $558(5)$ & $367(2)$ & $62(2)$ \\
\hline C (32) & $3778(6)$ & $1068(4)$ & $811(2)$ & $48(1)$ \\
\hline C (33) & $5519(7)$ & $-512(4)$ & $1170(2)$ & $62(2)$ \\
\hline C (34) & $6624(7)$ & $-718(5)$ & $1584(2)$ & $64(2)$ \\
\hline C (35) & $6889(6)$ & $-356(5)$ & $2389(2)$ & $64(2)$ \\
\hline C ( 41) & $7810(6)$ & $2160(5)$ & $474(2)$ & $57(2)$ \\
\hline C (42) & $6683(6)$ & $2675(4)$ & $154(2)$ & $49(1)$ \\
\hline C (43) & $7045(6)$ & $2658(4)$ & $-293(2)$ & $49(1)$ \\
\hline C ( 44$)$ & $8514(7)$ & $2141(5)$ & $-390(2)$ & $66(2)$ \\
\hline C ( 4 5) & $9506(7)$ & $1638(6)$ & $-74(3)$ & $76(2)$ \\
\hline$C(46)$ & 9165 (7) & $1661(6)$ & $362(2)$ & $71(2)$ \\
\hline C (51) & $2672(6)$ & $4162(4)$ & $-35(2)$ & $48(1)$ \\
\hline$C(52)$ & $4152(6)$ & $3629(4)$ & $-98(2)$ & $43(1)$ \\
\hline C (53) & $4525(6)$ & $3559(4)$ & $-544(2)$ & $48(1)$ \\
\hline C (54) & $3412(7)$ & $3975(5)$ & $-891(2)$ & $58(2)$ \\
\hline C (55) & $2004(8)$ & $4409(5)$ & $-813(2)$ & $65(2)$ \\
\hline$C(56)$ & $1660(7)$ & $4540(5)$ & $-374(2)$ & $61(2)$ \\
\hline
\end{tabular}


Table S3c. Bond lengths [A] and angles [deg] of trans$\left[\mathrm{Cr}(\mathrm{mac})(\mathrm{ONO})_{2}\right] \mathrm{BF}_{4}$ (III).

\begin{tabular}{|c|c|}
\hline $\operatorname{Cr}(1)-O(1)$ & $1.946(4)$ \\
\hline $\operatorname{Cr}(1)-O(3)$ & $1.968(3)$ \\
\hline $\operatorname{Cr}(1)-\mathrm{N}(4)$ & $2.058(4)$ \\
\hline $\operatorname{Cr}(1)-N(6)$ & $2.065(5)$ \\
\hline $\operatorname{Cr}(1)-\mathrm{N}(5)$ & $2.065(4)$ \\
\hline $\operatorname{Cr}(1)-\mathrm{N}(3)$ & $2.073(4)$ \\
\hline $\mathrm{O}(1)-\mathrm{N}(1)$ & $1.129(8)$ \\
\hline$B(1)-F(1)$ & $1.328(6)$ \\
\hline$B(1)-F(2)$ & $1.371(6)$ \\
\hline$B(1)-F(3)$ & $1.376(7)$ \\
\hline$B(1)-F(4)$ & $1.382(6)$ \\
\hline $\mathrm{N}(1)-\mathrm{O}(2)$ & $1.232(8)$ \\
\hline $\mathrm{N}(2)-\mathrm{O}(4)$ & $1.196(5)$ \\
\hline $\mathrm{N}(2)-\mathrm{O}(3)$ & $1.239(6)$ \\
\hline $\mathrm{N}(3)-\mathrm{C}(22)$ & $1.474(6)$ \\
\hline$N(3)-C(23)$ & $1.493(6)$ \\
\hline $\mathrm{N}(4)-\mathrm{C}(24)$ & $1.470(6)$ \\
\hline $\mathrm{N}(4)-\mathrm{C}(25)$ & $1.487(6)$ \\
\hline$N(5)-C(32)$ & $1.449(7)$ \\
\hline$N(5)-C(33)$ & $1.492(6)$ \\
\hline$N(6)-C(34)$ & $1.475(8)$ \\
\hline $\mathrm{N}(6)-\mathrm{C}(35)$ & $1.485(9)$ \\
\hline$C(11)-C(32)$ & $1.526(7)$ \\
\hline$C(11)-C(22)$ & $1.540(6)$ \\
\hline C (11) - C (15) & $1.563(6)$ \\
\hline$C(12)-C(25)$ & $1.496(8)$ \\
\hline$C(12)-C(35)$ & $1.511(8)$ \\
\hline$C(13)-C(52)$ & $1.402(7)$ \\
\hline$C(13)-C(42)$ & $1.433(7)$ \\
\hline$C(13)-C(15)$ & $1.522(6)$ \\
\hline$C(14)-C(43)$ & $1.374(8)$ \\
\hline$C(14)-C(53)$ & $1.392(7)$ \\
\hline$C(21)-C(22)$ & $1.531(6)$ \\
\hline$C(23)-C(24)$ & $1.506(6)$ \\
\hline $\mathrm{C}(31)-\mathrm{C}(32)$ & $1.510(7)$ \\
\hline C (33) -C (34) & $1.511(9)$ \\
\hline$C(41)-C(46)$ & $1.360(8)$ \\
\hline$C(41)-C(42)$ & $1.419(8)$ \\
\hline$C(42)-C(43)$ & $1.441(7)$ \\
\hline$C(43)-C(44)$ & $1.436(8)$ \\
\hline$C(44)-C(45)$ & $1.338(9)$ \\
\hline$C(45)-C(46)$ & $1.402(9)$ \\
\hline$C(51)-C(56)$ & $1.343(7)$ \\
\hline$C(51)-C(52)$ & $1.425(7)$ \\
\hline$C(52)-C(53)$ & $1.443(7)$ \\
\hline$C(53)-C(54)$ & $1.421(8)$ \\
\hline$C(54)-C(55)$ & $1.336(8)$ \\
\hline$C(55)-C(56)$ & $1.421(7)$ \\
\hline $\mathrm{O}(1)-\operatorname{Cr}(1)-\mathrm{C}$ & $175.8(2)$ \\
\hline
\end{tabular}




\begin{tabular}{|c|c|}
\hline $\mathrm{O}(1)-\mathrm{Cr}(1)-\mathrm{N}(4)$ & $90.9(2)$ \\
\hline $\mathrm{O}(3)-\mathrm{Cr}(1)-\mathrm{N}(4)$ & $87.4(2)$ \\
\hline$O(1)-\operatorname{Cr}(1)-N(6)$ & $93.3(2)$ \\
\hline$O(3)-\operatorname{Cr}(1)-N(6)$ & $90.6(2)$ \\
\hline$N(4)-\operatorname{Cr}(1)-N(6)$ & $93.5(2)$ \\
\hline $\mathrm{O}(1)-\mathrm{Cr}(1)-\mathrm{N}(5)$ & $87.6(2)$ \\
\hline $\mathrm{O}(3)-\mathrm{Cr}(1)-\mathrm{N}(5)$ & $94.2(2)$ \\
\hline$N(4)-\operatorname{Cr}(1)-N(5)$ & $178.4(2)$ \\
\hline$N(6)-C r(1)-N(5)$ & $86.0(2)$ \\
\hline $\mathrm{O}(1)-\mathrm{Cr}(1)-\mathrm{N}(3)$ & $86.8(2)$ \\
\hline $\mathrm{O}(3)-\mathrm{Cr}(1)-\mathrm{N}(3)$ & $89.3(2)$ \\
\hline$N(4)-\operatorname{Cr}(1)-N(3)$ & $86.0(2)$ \\
\hline$N(6)-C r(1)-N(3)$ & $179.5(2)$ \\
\hline$N(5)-\operatorname{Cr}(1)-N(3)$ & $94.5(2)$ \\
\hline$N(1)-O(1)-\operatorname{Cr}(1)$ & $135.6(6)$ \\
\hline $\mathrm{F}(1)-\mathrm{B}(1)-\mathrm{F}(2)$ & $112.6(5)$ \\
\hline$F(1)-B(1)-F(3)$ & $110.7(5)$ \\
\hline$F(2)-B(1)-F(3)$ & $104.3(4)$ \\
\hline $\mathrm{F}(1)-\mathrm{B}(1)-\mathrm{F}(4)$ & $110.7(4)$ \\
\hline$F(2)-B(1)-F(4)$ & $108.8(4)$ \\
\hline$F(3)-B(1)-F(4)$ & $109.4(4)$ \\
\hline $\mathrm{O}(1)-\mathrm{N}(1)-\mathrm{O}(2)$ & $122.1(8)$ \\
\hline $\mathrm{O}(4)-\mathrm{N}(2)-\mathrm{O}(3)$ & $118.1(5)$ \\
\hline$N(2)-O(3)-\operatorname{Cr}(1)$ & $121.7(3)$ \\
\hline$C(22)-N(3)-C(23)$ & $114.4(3)$ \\
\hline $\mathrm{C}(22)-\mathrm{N}(3)-\mathrm{Cr}(1)$ & $116.6(3)$ \\
\hline $\mathrm{C}(23)-\mathrm{N}(3)-\mathrm{Cr}(1)$ & $104.3(3)$ \\
\hline $\mathrm{C}(24)-\mathrm{N}(4)-\mathrm{C}(25)$ & $113.5(4)$ \\
\hline $\mathrm{C}(24)-\mathrm{N}(4)-\mathrm{Cr}(1)$ & $106.3(3)$ \\
\hline $\mathrm{C}(25)-\mathrm{N}(4)-\mathrm{Cr}(1)$ & $116.9(3)$ \\
\hline$C(32)-N(5)-C(33)$ & $116.9(4)$ \\
\hline$C(32)-N(5)-C r(1)$ & $117.3(3)$ \\
\hline $\mathrm{C}(33)-\mathrm{N}(5)-\mathrm{Cr}(1)$ & $106.0(3)$ \\
\hline$C(34)-N(6)-C(35)$ & $114.7(5)$ \\
\hline $\mathrm{C}(34)-\mathrm{N}(6)-\mathrm{Cr}(1)$ & $105.3(4)$ \\
\hline $\mathrm{C}(35)-\mathrm{N}(6)-\mathrm{Cr}(1)$ & $116.6(4)$ \\
\hline$C(32)-C(11)-C(22)$ & $113.7(4)$ \\
\hline$C(32)-C(11)-C(15)$ & $114.6(4)$ \\
\hline$C(22)-C(11)-C(15)$ & $113.6(4)$ \\
\hline$C(25)-C(12)-C(35)$ & $115.6(4)$ \\
\hline$C(52)-C(13)-C(42)$ & $119.5(4)$ \\
\hline$C(52)-C(13)-C(15)$ & $119.4(4)$ \\
\hline$C(42)-C(13)-C(15)$ & $121.1(5)$ \\
\hline$C(43)-C(14)-C(53)$ & $121.6(5)$ \\
\hline$C(13)-C(15)-C(11)$ & $115.8(4)$ \\
\hline $\mathrm{N}(3)-\mathrm{C}(22)-\mathrm{C}(21)$ & $112.5(4)$ \\
\hline $\mathrm{N}(3)-\mathrm{C}(22)-\mathrm{C}(11)$ & $112.8(4)$ \\
\hline$C(21)-C(22)-C(11)$ & $113.0(4)$ \\
\hline$N(3)-C(23)-C(24)$ & $109.5(4)$ \\
\hline$N(4)-C(24)-C(23)$ & $109.0(4)$ \\
\hline$N(4)-C(25)-C(12)$ & $112.3(5)$ \\
\hline$N(5)-C(32)-C(31)$ & $114.4(4)$ \\
\hline $\mathrm{N}(5)-\mathrm{C}(32)-\mathrm{C}(11)$ & $114.2(4)$ \\
\hline$C(31)-C(32)-C(11)$ & $114.5(4)$ \\
\hline
\end{tabular}




$\begin{array}{ll}N(5)-C(33)-C(34) & 108.9(4) \\ N(6)-C(34)-C(33) & 109.8(5) \\ N(6)-C(35)-C(12) & 113.7(5) \\ C(46)-C(41)-C(42) & 121.1(6) \\ C(41)-C(42)-C(13) & 124.1(5) \\ C(41)-C(42)-C(43) & 117.2(5) \\ C(13)-C(42)-C(43) & 118.6(5) \\ C(14)-C(43)-C(44) & 120.3(5) \\ C(14)-C(43)-C(42) & 120.7(5) \\ C(44)-C(43)-C(42) & 119.0(6) \\ C(45)-C(44)-C(43) & 120.9(6) \\ C(44)-C(45)-C(46) & 120.2(6) \\ C(41)-C(46)-C(45) & 121.5(6) \\ C(56)-C(51)-C(52) & 121.6(5) \\ C(13)-C(52)-C(51) & 123.2(4) \\ C(13)-C(52)-C(53) & 120.5(4) \\ C(51)-C(52)-C(53) & 116.3(5) \\ C(14)-C(53)-C(54) & 121.3(5) \\ C(14)-C(53)-C(52) & 119.0(5) \\ C(54)-C(53)-C(52) & 119.7(5) \\ C(55)-C(54)-C(53) & 121.2(5) \\ C(54)-C(55)-C(56) & 119.4(6) \\ C(51)-C(56)-C(55) & 121.5(5)\end{array}$

Symmetry transformations used to generate equivalent atoms: 
Table S3d. Anisotropic displacement parameters ( $A^{\wedge} 2 \times 10^{\wedge} 3$ ) of trans-[Cr (mac) (ONO) $\left.{ }_{2}\right] \mathrm{BF}_{4}$ (III). The anisotropic displacement factor exponent takes the form: $-2 \mathrm{pi}^{\wedge} 2\left[\mathrm{~h}^{\wedge} 2 \mathrm{a}^{\star \wedge} 2 \mathrm{U} 11+\ldots+2 \mathrm{~h} k \mathrm{a}^{\star} \mathrm{b}^{\star}\right.$ $\mathrm{U} 12$ ]

\begin{tabular}{|c|c|c|c|c|c|c|}
\hline & $\mathrm{U} 11$ & $\mathrm{U} 22$ & U33 & $\mathrm{U} 23$ & U13 & U12 \\
\hline $\operatorname{Cr}(1)$ & $29(1)$ & $19(1)$ & $64(1)$ & $1(1)$ & $7(1)$ & $-3(1)$ \\
\hline$O(1)$ & $53(2)$ & $58(3)$ & $119(3)$ & $-29(2)$ & $38(2)$ & $-32(2)$ \\
\hline$B(1)$ & $48(3)$ & $29(3)$ & $46(3)$ & $-3(2)$ & $7(3)$ & $0(2)$ \\
\hline$F(1)$ & $125(3)$ & $33(2)$ & $180(4)$ & $-21(2)$ & $68(3)$ & $-29(2)$ \\
\hline $\mathrm{N}(1)$ & $83(5)$ & $98(6)$ & $189(8)$ & $22(5)$ & $14(5)$ & $-21(5)$ \\
\hline$F(2)$ & $107(3)$ & $85(3)$ & 77 (2) & $7(2)$ & $39(2)$ & 27 (2) \\
\hline $\mathrm{N}(2)$ & $47(3)$ & $55(3)$ & $99(4)$ & $1(3)$ & $4(3)$ & $-20(2)$ \\
\hline$O(2)$ & $52(3)$ & $86(4)$ & $167(5)$ & $27(3)$ & $-13(3)$ & $-39(3)$ \\
\hline$O(3)$ & $33(2)$ & $30(2)$ & $73(2)$ & $-5(2)$ & $8(2)$ & $-5(1)$ \\
\hline $\mathrm{N}(3)$ & $28(2)$ & $27(2)$ & $47(2)$ & $-5(2)$ & $6(2)$ & $-5(2)$ \\
\hline F (3) & $80(3)$ & $102(3)$ & $124(3)$ & $-44(3)$ & $-10(2)$ & $30(2)$ \\
\hline$F(4)$ & $60(2)$ & $37(2)$ & $74(2)$ & $-3(1)$ & $26(2)$ & $0(1)$ \\
\hline N (4) & $36(2)$ & $40(3)$ & $59(3)$ & $14(2)$ & $2(2)$ & $-6(2)$ \\
\hline$O(4)$ & $49(2)$ & $66(3)$ & $102(3)$ & $-10(2)$ & $14(2)$ & $-21(2)$ \\
\hline$N(5)$ & $32(2)$ & $27(2)$ & $79(3)$ & $-16(2)$ & $15(2)$ & $-8(2)$ \\
\hline$N(6)$ & $44(3)$ & $24(2)$ & $104(4)$ & $9(2)$ & $17(3)$ & $-4(2)$ \\
\hline$C(11)$ & $35(2)$ & $34(3)$ & $48(3)$ & $-9(2)$ & $9(2)$ & $-12(2)$ \\
\hline C (12) & $44(3)$ & $58(4)$ & $78(4)$ & $33(3)$ & $5(3)$ & $-3(3)$ \\
\hline C (13) & $42(3)$ & $26(2)$ & $54(3)$ & $-7(2)$ & $15(2)$ & $-13(2)$ \\
\hline$C(14)$ & $63(4)$ & $34(3)$ & $61(3)$ & $-12(2)$ & $29(3)$ & $-19(2)$ \\
\hline C (15) & $43(3)$ & $24(2)$ & $60(3)$ & $-4(2)$ & $10(2)$ & $-8(2)$ \\
\hline C (21) & $46(3)$ & $46(3)$ & $44(3)$ & $1(2)$ & $5(2)$ & $10(2)$ \\
\hline$C(22)$ & $34(2)$ & $32(2)$ & $42(2)$ & $-6(2)$ & $9(2)$ & $-3(2)$ \\
\hline C (23) & $34(2)$ & $32(2)$ & $48(3)$ & $-3(2)$ & $10(2)$ & $1(2)$ \\
\hline C (24) & $42(3)$ & $40(3)$ & $46(3)$ & $-2(2)$ & $6(2)$ & $-4(2)$ \\
\hline C (25) & $38(3)$ & $60(4)$ & $60(3)$ & $20(3)$ & $-3(2)$ & $-2(2)$ \\
\hline C (31) & $65(4)$ & $47(3)$ & $81(4)$ & $-35(3)$ & $29(3)$ & $-24(3)$ \\
\hline C (32) & $62(3)$ & $30(3)$ & $56(3)$ & $-12(2)$ & $26(3)$ & $-15(2)$ \\
\hline C (33) & $63(4)$ & $23(3)$ & $107(4)$ & $-16(3)$ & $37(3)$ & $-5(2)$ \\
\hline C (34) & $47(3)$ & $24(3)$ & $122(5)$ & $6(3)$ & $18(3)$ & $3(2)$ \\
\hline C (35) & $43(3)$ & $38(3)$ & $107(5)$ & $25(3)$ & $-6(3)$ & $2(2)$ \\
\hline C (41) & $42(3)$ & $52(3)$ & $78(4)$ & $-22(3)$ & $6(3)$ & $-17(3)$ \\
\hline C (42) & $42(3)$ & $33(3)$ & $72(3)$ & $-14(2)$ & $17(3)$ & $-22(2)$ \\
\hline$C(43)$ & $43(3)$ & $35(3)$ & $74(3)$ & $-21(3)$ & $28(3)$ & $-21(2)$ \\
\hline C ( 44$)$ & $49(3)$ & $59(4)$ & $94(4)$ & $-31(3)$ & $29(3)$ & $-21(3)$ \\
\hline C (45) & $44(4)$ & $79(5)$ & $108(5)$ & $-41(4)$ & $20(4)$ & $-11(3)$ \\
\hline$C(46)$ & $36(3)$ & $71(4)$ & $104(5)$ & $-24(4)$ & $-2(3)$ & $-8(3)$ \\
\hline C (51) & $61(3)$ & $35(3)$ & $50(3)$ & $4(2)$ & $21(3)$ & $-2(2)$ \\
\hline C (52) & $49(3)$ & $29(3)$ & $52(3)$ & $-5(2)$ & $19(2)$ & $-15(2)$ \\
\hline C (53) & $64(3)$ & $30(3)$ & $55(3)$ & $-1(2)$ & $25(3)$ & $-13(2)$ \\
\hline$C(54)$ & $86(4)$ & $43(3)$ & $50(3)$ & $2(2)$ & $25(3)$ & $-5(3)$ \\
\hline C (55) & $78(4)$ & $67(4)$ & $51(3)$ & $7(3)$ & $16(3)$ & $16(3)$ \\
\hline$C(56)$ & 77 (4) & $54(3)$ & $55(3)$ & $11(3)$ & $22(3)$ & $12(3)$ \\
\hline
\end{tabular}




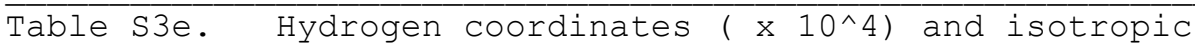

displacement parameters $\left(A^{\wedge} 2 \times 10^{\wedge} 3\right)$ of $\operatorname{trans}-\left[\mathrm{Cr}(\operatorname{mac})(\mathrm{ONO})_{2}\right] \mathrm{BF}_{4}$ (III).

\begin{tabular}{|c|c|c|c|c|}
\hline & $x$ & $\mathrm{Y}$ & $\mathrm{z}$ & $\mathrm{U}(\mathrm{eq})$ \\
\hline $\mathrm{H}(3)$ & $4128(50)$ & $3162(37)$ & $1597(13)$ & $13(12)$ \\
\hline $\mathrm{H}(4)$ & $3677(56)$ & $1198(42)$ & $2454(14)$ & $28(13)$ \\
\hline $\mathrm{H}(5)$ & $5843(74)$ & $1064(53)$ & 1091 (18) & $66(18)$ \\
\hline $\mathrm{H}(6)$ & $5270(92)$ & $-542(74)$ & $1971(28)$ & $80(32)$ \\
\hline $\mathrm{H}(11)$ & $2557(5)$ & $2455(4)$ & $550(2)$ & 46 \\
\hline $\mathrm{H}(12 \mathrm{~A})$ & $5114(6)$ & $-403(5)$ & $2785(2)$ & 72 \\
\hline $\mathrm{H}(12 \mathrm{~B})$ & $6769(6)$ & $-101(5)$ & $3040(2)$ & 72 \\
\hline $\mathrm{H}(14)$ & $6265(7)$ & $3108(4)$ & $-909(2)$ & 61 \\
\hline $\mathrm{H}(15 \mathrm{~A})$ & $4439(6)$ & $3995(4)$ & $774(2)$ & 50 \\
\hline $\mathrm{H}(15 \mathrm{~B})$ & $5673(6)$ & $3006(4)$ & $910(2)$ & 50 \\
\hline $\mathrm{H}(21 \mathrm{~A})$ & $943(29)$ & $3877(11)$ & $839(5)$ & 68 \\
\hline $\mathrm{H}(21 \mathrm{~B})$ & $2351(6)$ & $4540(5)$ & $1109(10)$ & 68 \\
\hline $\mathrm{H}(21 \mathrm{C})$ & $945(30)$ & $4046(14)$ & 1345 ( 6$)$ & 68 \\
\hline $\mathrm{H}(22)$ & $1679(5)$ & 2161 (4) & $1226(1)$ & 43 \\
\hline $\mathrm{H}(23 \mathrm{~A})$ & $1768(5)$ & $2613(4)$ & $2006(1)$ & 45 \\
\hline $\mathrm{H}(23 \mathrm{~B})$ & $2443(5)$ & $3891(4)$ & $1989(1)$ & 45 \\
\hline $\mathrm{H}(24 \mathrm{~A})$ & $4765(6)$ & $3335(4)$ & $2422(2)$ & 51 \\
\hline $\mathrm{H}(24 \mathrm{~B})$ & $3298(6)$ & $3008(4)$ & $2669(2)$ & 51 \\
\hline $\mathrm{H}(25 \mathrm{~A})$ & $5302(6)$ & $1533(5)$ & $3052(2)$ & 64 \\
\hline $\mathrm{H}(25 \mathrm{~B})$ & 6555 ( 6 ) & $1790(5)$ & $2725(2)$ & 64 \\
\hline $\mathrm{H}(31 \mathrm{~A})$ & $3166(32)$ & $869(28)$ & $153(3)$ & 94 \\
\hline $\mathrm{H}(31 \mathrm{~B})$ & $3869(50)$ & $-278(6)$ & $378(4)$ & 94 \\
\hline $\mathrm{H}(31 \mathrm{C})$ & $5003(20)$ & $757(32)$ & $287(6)$ & 94 \\
\hline $\mathrm{H}(32)$ & $2823(6)$ & $683(4)$ & $895(2)$ & 57 \\
\hline $\mathrm{H}(33 \mathrm{~A})$ & $6061(7)$ & $-697(4)$ & $916(2)$ & 75 \\
\hline $\mathrm{H}(33 \mathrm{~B})$ & $4593(7)$ & $-1014(4)$ & $1165(2)$ & 75 \\
\hline $\mathrm{H}(34 \mathrm{~A})$ & $6904(7)$ & $-1539(5)$ & $1608(2)$ & 76 \\
\hline $\mathrm{H}(34 \mathrm{~B})$ & $7594(7)$ & $-271(5)$ & $1575(2)$ & 76 \\
\hline $\mathrm{H}(35 \mathrm{~A})$ & $7795(6)$ & $145(5)$ & $2360(2)$ & 77 \\
\hline $\mathrm{H}(35 \mathrm{~B})$ & $7287(6)$ & $-1142(5)$ & $2448(2)$ & 77 \\
\hline $\mathrm{H}(41)$ & $7618(6)$ & $2164(5)$ & $766(2)$ & 69 \\
\hline $\mathrm{H}(44)$ & $8779(7)$ & $2156(5)$ & $-675(2)$ & 79 \\
\hline $\mathrm{H}(45)$ & $10426(7)$ & $1272(6)$ & $-144(3)$ & 91 \\
\hline $\mathrm{H}(46)$ & $9881(7)$ & $1327(6)$ & $578(2)$ & 85 \\
\hline $\mathrm{H}(51)$ & $2400(6)$ & $4249(4)$ & $248(2)$ & 57 \\
\hline $\mathrm{H}(54)$ & $3668(7)$ & $3945(5)$ & $-1177(2)$ & 70 \\
\hline $\mathrm{H}(55)$ & $1256(8)$ & $4623(5)$ & $-1045(2)$ & 78 \\
\hline $\mathrm{H}(56)$ & $713(7)$ & $4895(5)$ & $-320(2)$ & 73 \\
\hline
\end{tabular}


Table S4a. Crystal data and structure refinement trans$\left[\mathrm{Cr}(\mathrm{hbc})(\mathrm{ONO})_{2}\right] \mathrm{BF}_{4} \cdot$ Acetone (IV).

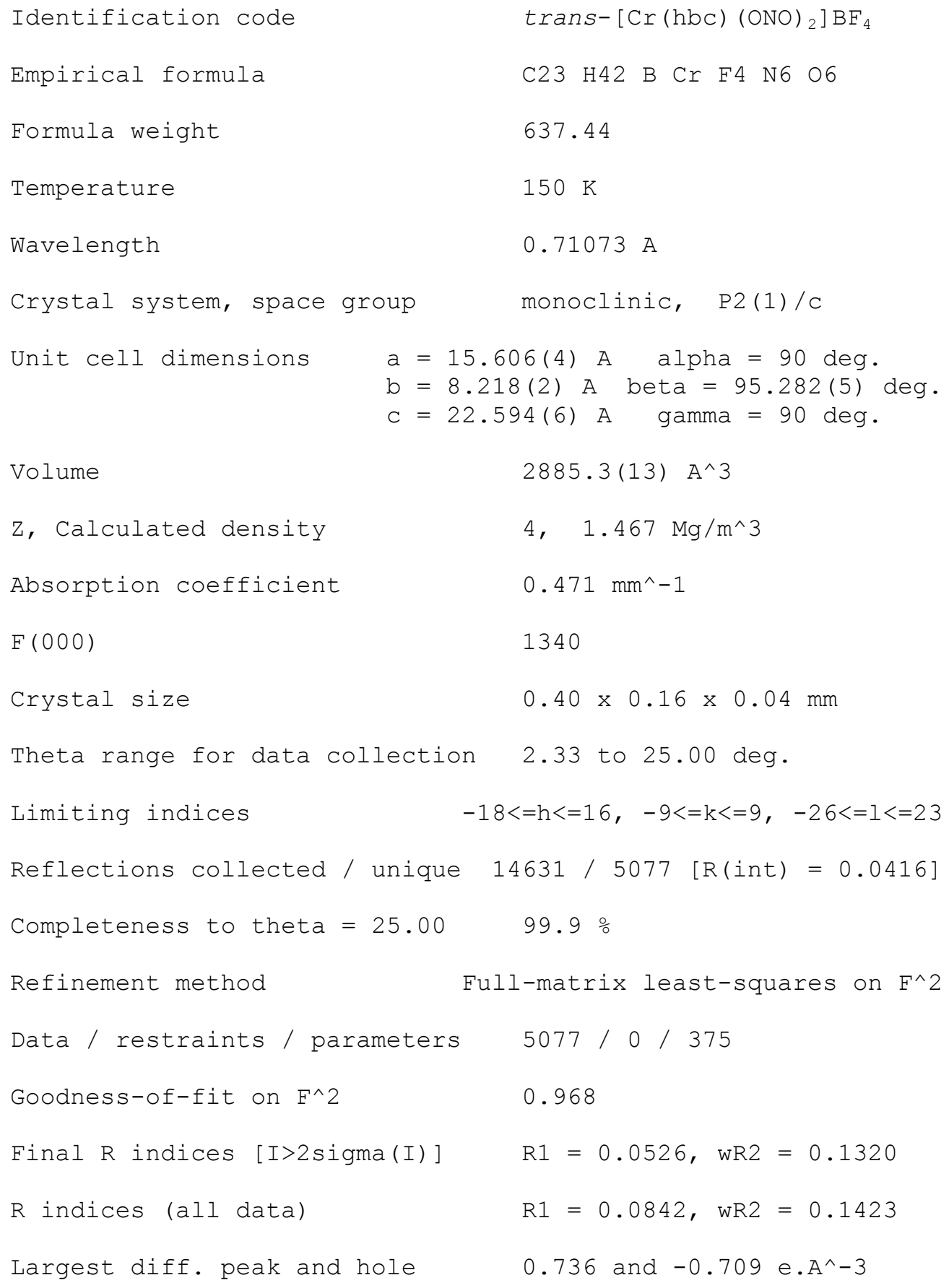

Volume

Z, Calculated density

Absorption coefficient

F ( 000$)$

Crystal size

Theta range for data collection

$2885.3(13) \quad A^{\wedge} 3$

4, $1.467 \mathrm{Mg} / \mathrm{m}^{\wedge} 3$

$0.471 \mathrm{~mm}^{\wedge}-1$

1340

$0.40 \times 0.16 \times 0.04 \mathrm{~mm}$

2.33 to 25.00 deg.

Limiting indices

Reflections collected / unique

Completeness to theta $=25.00$

Refinement method

Data / restraints / parameters

Goodness-of-fit on $\mathrm{F}^{\wedge} 2$

Final $R$ indices [I>2sigma(I)]

$\mathrm{R}$ indices (all data)

Largest diff. peak and hole

$-18<=\mathrm{h}<=16, \quad-9<=\mathrm{k}<=9, \quad-26<=1<=23$

$14631 / 5077$ [R(int) $=0.0416]$

$99.9 \%$

Full-matrix least-squares on $\mathrm{F}^{\wedge} 2$

$5077 / 0 / 375$

0.968

$\mathrm{R} 1=0.0526, \mathrm{wR} 2=0.1320$

$\mathrm{R} 1=0.0842, \mathrm{wR} 2=0.1423$

0.736 and -0.709 e. $A^{\wedge}-3$ 
Table S4b. Atomic coordinates $\left(x 1^{\wedge} 4\right)$ and equivalent isotropic displacement parameters $\left(A^{\wedge} 2 \times 10^{\wedge} 3\right)$ trans-

$\left[\mathrm{Cr}(\mathrm{hbc})(\mathrm{ONO})_{2}\right] \mathrm{BF}_{4}$. Acetone (IV). U(eq) is defined as one third of the trace of the orthogonalized Uij tensor.

\begin{tabular}{|c|c|c|c|c|}
\hline & $x$ & $\mathrm{y}$ & z & $\mathrm{U}(\mathrm{eq})$ \\
\hline $\operatorname{Cr}(1)$ & $3390(1)$ & $1519(1)$ & $1270(1)$ & $26(1)$ \\
\hline$O(1)$ & $3134(2)$ & $-670(3)$ & $949(1)$ & $36(1)$ \\
\hline C (1) & $1504(2)$ & $675(4)$ & $1841(2)$ & $36(1)$ \\
\hline$F(1)$ & $5251(2)$ & $7360(4)$ & $1465(1)$ & $82(1)$ \\
\hline B (1) & $6093(3)$ & $7343(5)$ & $1316(2)$ & $38(1)$ \\
\hline $\mathrm{N}(1)$ & $3502(2)$ & $-1266(4)$ & $509(2)$ & $54(1)$ \\
\hline$O(2)$ & $3270(2)$ & $-2621(3)$ & $361(1)$ & $62(1)$ \\
\hline C (2) & $5259(2)$ & $2311(5)$ & $686(2)$ & $45(1)$ \\
\hline F (2) & $6310(2)$ & $5795(3)$ & $1147(1)$ & $67(1)$ \\
\hline $\mathrm{N}(2)$ & $3029(2)$ & $4542(4)$ & $1809(2)$ & $60(1)$ \\
\hline$O(3)$ & $3652(1)$ & $3663(3)$ & $1634(1)$ & $35(1)$ \\
\hline $\mathrm{N}(3)$ & $3113(2)$ & $610(3)$ & $2082(1)$ & $30(1)$ \\
\hline F (3) & $6605(2)$ & $7825(3)$ & $1813(1)$ & $65(1)$ \\
\hline $\mathrm{N}(4)$ & $4647(2)$ & $868(3)$ & $1523(1)$ & $32(1)$ \\
\hline$O(4)$ & $3246(2)$ & $5765(3)$ & $2069(1)$ & $59(1)$ \\
\hline $\mathrm{F}(4)$ & $6150(2)$ & $8423(3)$ & $854(1)$ & $70(1)$ \\
\hline$N(5)$ & $2126(2)$ & $2150(3)$ & $1004(1)$ & $32(1)$ \\
\hline$O(5)$ & $-1526(4)$ & $930(7)$ & $4072(2)$ & $148(2)$ \\
\hline $\mathrm{N}(6)$ & $3676(2)$ & $2417(3)$ & $457(1)$ & $32(1)$ \\
\hline$O(6)$ & $8607(2)$ & $1121(4)$ & $253(1)$ & $71(1)$ \\
\hline$C(11)$ & $778(2)$ & $2048(4)$ & $2701(2)$ & $41(1)$ \\
\hline$C(12)$ & $1083(3)$ & $2493(5)$ & $3271(2)$ & $50(1)$ \\
\hline$C(13)$ & $570(3)$ & $2353(5)$ & $3746(2)$ & $57(1)$ \\
\hline$C(14)$ & $-266(3)$ & $1754(5)$ & $3653(2)$ & $51(1)$ \\
\hline$C(15)$ & $-576(3)$ & $1341(5)$ & $3081(2)$ & $50(1)$ \\
\hline$C(16)$ & $-65(2)$ & $1472(4)$ & $2612(2)$ & $43(1)$ \\
\hline$C(17)$ & $1336(2)$ & $2244(4)$ & $2194(2)$ & $42(1)$ \\
\hline$C(18)$ & $-763(4)$ & $1576(7)$ & $4186(3)$ & $89(2)$ \\
\hline$C(21)$ & $2183(2)$ & $-940(4)$ & $2725(2)$ & $40(1)$ \\
\hline$C(22)$ & $2287(2)$ & $-313(4)$ & $2099(2)$ & $33(1)$ \\
\hline C (23) & $3894(2)$ & $-344(4)$ & $2311(2)$ & $36(1)$ \\
\hline C (24) & $4687(2)$ & $583(5)$ & $2173(2)$ & $37(1)$ \\
\hline$C(25)$ & $5317(2)$ & $2010(5)$ & $1351(2)$ & $41(1)$ \\
\hline C (31) & $561(2)$ & $1424(6)$ & $900(2)$ & $56(1)$ \\
\hline C (32) & $1467(2)$ & $957(5)$ & $1161(2)$ & $38(1)$ \\
\hline C (33) & $2105(2)$ & $2485(5)$ & $352(2)$ & $39(1)$ \\
\hline C (34) & $2916(2)$ & $3388(4)$ & $232(2)$ & $40(1)$ \\
\hline$C(35)$ & $4491(2)$ & $3324(5)$ & $444(2)$ & $42(1)$ \\
\hline$C(41)$ & $8398(4)$ & $3586(6)$ & $725(2)$ & $76(2)$ \\
\hline C (42) & $8260(3)$ & $1832(5)$ & $629(2)$ & $50(1)$ \\
\hline$C(43)$ & $7717(3)$ & $1005(7)$ & $1025(2)$ & $79(2)$ \\
\hline
\end{tabular}


Table S4c. Bond lengths [A] and angles [deg] of trans-[Cr $\left.(\mathrm{hbc})(\mathrm{ONO})_{2}\right] \mathrm{BF}_{4}$. Acetone (IV) .

\begin{tabular}{|c|c|}
\hline $\operatorname{Cr}(1)-O(1)$ & $1.968(2)$ \\
\hline $\operatorname{Cr}(1)-O(3)$ & $1.971(2)$ \\
\hline $\operatorname{Cr}(1)-N(4)$ & $2.062(3)$ \\
\hline $\operatorname{Cr}(1)-N(3)$ & $2.064(3)$ \\
\hline $\operatorname{Cr}(1)-N(6)$ & $2.065(3)$ \\
\hline $\operatorname{Cr}(1)-N(5)$ & $2.075(3)$ \\
\hline $\mathrm{O}(1)-\mathrm{N}(1)$ & $1.290(4)$ \\
\hline$C(1)-C(22)$ & $1.536(5)$ \\
\hline$C(1)-C(32)$ & $1.549(5)$ \\
\hline$C(1)-C(17)$ & $1.551(5)$ \\
\hline$F(1)-B(1)$ & $1.387(5)$ \\
\hline$B(1)-F(3)$ & $1.376(5)$ \\
\hline$B(1)-F(2)$ & $1.379(5)$ \\
\hline$B(1)-F(4)$ & $1.380(5)$ \\
\hline $\mathrm{N}(1)-\mathrm{O}(2)$ & $1.209(4)$ \\
\hline$C(2)-C(25)$ & $1.517(5)$ \\
\hline$C(2)-C(35)$ & $1.519(5)$ \\
\hline$N(2)-O(4)$ & $1.196(4)$ \\
\hline $\mathrm{N}(2)-\mathrm{O}(3)$ & $1.301(4)$ \\
\hline$N(3)-C(22)$ & $1.500(4)$ \\
\hline$N(3)-C(23)$ & $1.500(4)$ \\
\hline$N(4)-C(24)$ & $1.483(4)$ \\
\hline$N(4)-C(25)$ & $1.483(4)$ \\
\hline$N(5)-C(32)$ & $1.487(4)$ \\
\hline$N(5)-C(33)$ & $1.497(4)$ \\
\hline $\mathrm{O}(5)-\mathrm{C}(18)$ & $1.308(7)$ \\
\hline$N(6)-C(35)$ & $1.477(4)$ \\
\hline$N(6)-C(34)$ & $1.479(4)$ \\
\hline$O(6)-C(42)$ & $1.201(5)$ \\
\hline$C(11)-C(12)$ & $1.381(5)$ \\
\hline$C(11)-C(16)$ & $1.396(5)$ \\
\hline$C(11)-C(17)$ & $1.510(5)$ \\
\hline$C(12)-C(13)$ & $1.402(5)$ \\
\hline$C(13)-C(14)$ & $1.392(6)$ \\
\hline$C(14)-C(15)$ & $1.381(6)$ \\
\hline$C(14)-C(18)$ & $1.500(6)$ \\
\hline$C(15)-C(16)$ & $1.387(5)$ \\
\hline$C(21)-C(22)$ & $1.528(5)$ \\
\hline$C(23)-C(24)$ & $1.510(5)$ \\
\hline$C(31)-C(32)$ & $1.531(5)$ \\
\hline$C(33)-C(34)$ & $1.514(5)$ \\
\hline$C(41)-C(42)$ & $1.470(6)$ \\
\hline$C(42)-C(43)$ & $1.457(6)$ \\
\hline$O(1)-\operatorname{Cr}(1)-O(3)$ & $176.97(10)$ \\
\hline $\mathrm{O}(1)-\mathrm{Cr}(1)-\mathrm{N}(4)$ & $91.20(11)$ \\
\hline $\mathrm{O}(3)-\mathrm{Cr}(1)-\mathrm{N}(4)$ & $87.79(10)$ \\
\hline $\mathrm{O}(1)-\mathrm{Cr}(1)-\mathrm{N}(3)$ & $86.83(10)$ \\
\hline $\mathrm{O}(3)-\mathrm{Cr}(1)-\mathrm{N}(3)$ & $90.25(10)$ \\
\hline $\mathrm{N}(4)-\mathrm{Cr}(1)-\mathrm{N}(3)$ & $86.08(10)$ \\
\hline $\mathrm{O}(1)-\mathrm{Cr}(1)-\mathrm{N}(6)$ & $92.99(10)$ \\
\hline $\mathrm{O}(3)-\operatorname{Cr}(1)-\mathrm{N}(6)$ & $89.92(10)$ \\
\hline
\end{tabular}




\begin{tabular}{|c|c|}
\hline$N(4)-\operatorname{Cr}(1)-N(6)$ & $93.54(11)$ \\
\hline$N(3)-C r(1)-N(6)$ & $179.58(11)$ \\
\hline $\mathrm{O}(1)-\mathrm{Cr}(1)-\mathrm{N}(5)$ & $88.03(11)$ \\
\hline $\mathrm{O}(3)-\mathrm{Cr}(1)-\mathrm{N}(5)$ & $93.00(10)$ \\
\hline $\mathrm{N}(4)-\mathrm{Cr}(1)-\mathrm{N}(5)$ & $179.09(11)$ \\
\hline$N(3)-C r(1)-N(5)$ & $94.36(10)$ \\
\hline$N(6)-C r(1)-N(5)$ & $86.01(11)$ \\
\hline $\mathrm{N}(1)-\mathrm{O}(1)-\mathrm{Cr}(1)$ & $122.8(2)$ \\
\hline$C(22)-C(1)-C(32)$ & $114.2(3)$ \\
\hline$C(22)-C(1)-C(17)$ & $114.2(3)$ \\
\hline$C(32)-C(1)-C(17)$ & $113.2(3)$ \\
\hline$F(3)-B(1)-F(2)$ & $110.6(3)$ \\
\hline $\mathrm{F}(3)-\mathrm{B}(1)-\mathrm{F}(4)$ & $111.1(3)$ \\
\hline $\mathrm{F}(2)-\mathrm{B}(1)-\mathrm{F}(4)$ & $110.5(3)$ \\
\hline $\mathrm{F}(3)-\mathrm{B}(1)-\mathrm{F}(1)$ & $106.9(3)$ \\
\hline $\mathrm{F}(2)-\mathrm{B}(1)-\mathrm{F}(1)$ & $109.8(3)$ \\
\hline $\mathrm{F}(4)-\mathrm{B}(1)-\mathrm{F}(1)$ & $107.8(3)$ \\
\hline $\mathrm{O}(2)-\mathrm{N}(1)-\mathrm{O}(1)$ & $114.8(3)$ \\
\hline$C(25)-C(2)-C(35)$ & $114.7(3)$ \\
\hline $\mathrm{O}(4)-\mathrm{N}(2)-\mathrm{O}(3)$ & $115.4(3)$ \\
\hline $\mathrm{N}(2)-\mathrm{O}(3)-\mathrm{Cr}(1)$ & $119.4(2)$ \\
\hline$C(22)-N(3)-C(23)$ & $113.6(2)$ \\
\hline $\mathrm{C}(22)-\mathrm{N}(3)-\mathrm{Cr}(1)$ & $117.13(19)$ \\
\hline $\mathrm{C}(23)-\mathrm{N}(3)-\mathrm{Cr}(1)$ & $105.35(18)$ \\
\hline $\mathrm{C}(24)-\mathrm{N}(4)-\mathrm{C}(25)$ & $113.2(3)$ \\
\hline $\mathrm{C}(24)-\mathrm{N}(4)-\mathrm{Cr}(1)$ & $105.54(19)$ \\
\hline $\mathrm{C}(25)-\mathrm{N}(4)-\mathrm{Cr}(1)$ & $116.0(2)$ \\
\hline $\mathrm{C}(32)-\mathrm{N}(5)-\mathrm{C}(33)$ & $113.8(3)$ \\
\hline $\mathrm{C}(32)-\mathrm{N}(5)-\mathrm{Cr}(1)$ & $115.2(2)$ \\
\hline $\mathrm{C}(33)-\mathrm{N}(5)-\mathrm{Cr}(1)$ & $105.4(2)$ \\
\hline $\mathrm{C}(35)-\mathrm{N}(6)-\mathrm{C}(34)$ & $112.7(3)$ \\
\hline $\mathrm{C}(35)-\mathrm{N}(6)-\mathrm{Cr}(1)$ & $117.0(2)$ \\
\hline $\mathrm{C}(34)-\mathrm{N}(6)-\mathrm{Cr}(1)$ & $105.49(19)$ \\
\hline$C(12)-C(11)-C(16)$ & $117.6(3)$ \\
\hline$C(12)-C(11)-C(17)$ & $120.4(4)$ \\
\hline$C(16)-C(11)-C(17)$ & $122.0(4)$ \\
\hline$C(11)-C(12)-C(13)$ & $121.4(4)$ \\
\hline$C(14)-C(13)-C(12)$ & $120.4(4)$ \\
\hline$C(15)-C(14)-C(13)$ & $118.2(4)$ \\
\hline$C(15)-C(14)-C(18)$ & $124.4(4)$ \\
\hline$C(13)-C(14)-C(18)$ & $117.4(4)$ \\
\hline$C(14)-C(15)-C(16)$ & $121.2(4)$ \\
\hline$C(15)-C(16)-C(11)$ & $121.2(4)$ \\
\hline$C(11)-C(17)-C(1)$ & $116.0(3)$ \\
\hline $\mathrm{O}(5)-\mathrm{C}(18)-\mathrm{C}(14)$ & $114.2(5)$ \\
\hline $\mathrm{N}(3)-\mathrm{C}(22)-\mathrm{C}(21)$ & $111.0(3)$ \\
\hline $\mathrm{N}(3)-\mathrm{C}(22)-\mathrm{C}(1)$ & $112.3(3)$ \\
\hline$C(21)-C(22)-C(1)$ & $112.4(3)$ \\
\hline$N(3)-C(23)-C(24)$ & $108.7(3)$ \\
\hline $\mathrm{N}(4)-\mathrm{C}(24)-\mathrm{C}(23)$ & $108.9(3)$ \\
\hline$N(4)-C(25)-C(2)$ & $112.6(3)$ \\
\hline $\mathrm{N}(5)-\mathrm{C}(32)-\mathrm{C}(31)$ & $112.2(3)$ \\
\hline $\mathrm{N}(5)-\mathrm{C}(32)-\mathrm{C}(1)$ & $111.8(3)$ \\
\hline$C(31)-C(32)-C(1)$ & $111.6(3)$ \\
\hline$N(5)-C(33)-C(34)$ & $108.9(3)$ \\
\hline$N(6)-C(34)-C(33)$ & $109.4(3)$ \\
\hline$N(6)-C(35)-C(2)$ & $111.7(3)$ \\
\hline
\end{tabular}




$\begin{array}{ll}O(6)-C(42)-C(43) & 122.3(4) \\ O(6)-C(42)-C(41) & 120.8(4) \\ C(43)-C(42)-C(41) & 116.9(4)\end{array}$

Symmetry transformations used to generate equivalent atoms: 
Table S4d. Anisotropic displacement parameters ( $\left.A^{\wedge} 2 \times 10^{\wedge} 3\right)$ of trans$\left[\mathrm{Cr}(\mathrm{hbc})(\mathrm{ONO})_{2}\right] \mathrm{BF}_{4}$. Acetone (IV). The anisotropic displacement factor exponent takes the form: $-2 \mathrm{pi}^{\wedge} 2\left[\mathrm{~h}^{\wedge} 2 \mathrm{a}^{\star \wedge} 2 \mathrm{U} 11+\ldots+2 \mathrm{~h} \mathrm{k} \mathrm{a}^{\star} \mathrm{b} \mathrm{b}^{\star} \mathrm{U} 12\right.$ ]

\begin{tabular}{|c|c|c|c|c|c|c|}
\hline & U11 & U22 & U33 & U23 & U13 & U12 \\
\hline $\operatorname{Cr}(1)$ & 31 (1) & $25(1)$ & $24(1)$ & $0(1)$ & $3(1)$ & $-1(1)$ \\
\hline$O(1)$ & $46(1)$ & $28(1)$ & $34(1)$ & $-8(1)$ & $7(1)$ & $0(1)$ \\
\hline $\mathrm{C}(1)$ & $33(2)$ & $35(2)$ & $40(2)$ & $4(2)$ & $9(2)$ & $0(2)$ \\
\hline$F(1)$ & $58(2)$ & $92(2)$ & $98(2)$ & $5(2)$ & $20(2)$ & $5(2)$ \\
\hline B (1) & $51(3)$ & $35(2)$ & $30(2)$ & $1(2)$ & $5(2)$ & $0(2)$ \\
\hline $\mathrm{N}(1)$ & $76(2)$ & $40(2)$ & $50(2)$ & $-8(2)$ & $21(2)$ & $-6(2)$ \\
\hline$O(2)$ & $100(2)$ & $31(2)$ & $57(2)$ & $-14(1)$ & $23(2)$ & $-9(2)$ \\
\hline$C(2)$ & $40(2)$ & $53(2)$ & $43(2)$ & $-8(2)$ & $16(2)$ & $-12(2)$ \\
\hline$F(2)$ & $106(2)$ & $45(1)$ & $53(2)$ & $-1(1)$ & $15(1)$ & $16(1)$ \\
\hline $\mathrm{N}(2)$ & $64(2)$ & $37(2)$ & $81(3)$ & $-23(2)$ & $23(2)$ & $-7(2)$ \\
\hline$O(3)$ & $38(1)$ & $28(1)$ & $39(1)$ & $-6(1)$ & $7(1)$ & $-1(1)$ \\
\hline$N(3)$ & $35(2)$ & $28(2)$ & $28(2)$ & $0(1)$ & $2(1)$ & $0(1)$ \\
\hline$F(3)$ & $86(2)$ & $64(2)$ & $41(1)$ & $2(1)$ & $-12(1)$ & $-8(1)$ \\
\hline$N(4)$ & $30(2)$ & $35(2)$ & $29(2)$ & $-4(1)$ & $2(1)$ & $1(1)$ \\
\hline$O(4)$ & $70(2)$ & $33(2)$ & $74(2)$ & $-14(2)$ & $11(2)$ & $-2(1)$ \\
\hline$F(4)$ & $123(2)$ & $46(1)$ & $39(1)$ & $8(1)$ & $5(1)$ & $-12(2)$ \\
\hline$N(5)$ & $35(2)$ & $34(2)$ & $27(2)$ & $3(1)$ & $2(1)$ & $2(1)$ \\
\hline$O(5)$ & $210(6)$ & $138(4)$ & $109(4)$ & $-28(3)$ & $92(4)$ & $-58(4)$ \\
\hline$N(6)$ & $42(2)$ & $31(2)$ & $25(1)$ & $0(1)$ & $6(1)$ & $-3(1)$ \\
\hline$O(6)$ & $68(2)$ & $84(2)$ & $61(2)$ & $-24(2)$ & $16(2)$ & $-12(2)$ \\
\hline$C(11)$ & $38(2)$ & $31(2)$ & $55(2)$ & $0(2)$ & $14(2)$ & $5(2)$ \\
\hline $\mathrm{C}(12)$ & $43(2)$ & $46(3)$ & $62(3)$ & $-8(2)$ & $8(2)$ & $2(2)$ \\
\hline$C(13)$ & $68(3)$ & $56(3)$ & $47(3)$ & $-8(2)$ & $10(2)$ & $4(2)$ \\
\hline $\mathrm{C}(14)$ & $66(3)$ & $35(2)$ & $57(3)$ & $1(2)$ & $27(2)$ & $7(2)$ \\
\hline$C(15)$ & $38(2)$ & $35(2)$ & $79(3)$ & $8(2)$ & $23(2)$ & $0(2)$ \\
\hline$C(16)$ & $43(2)$ & $37(2)$ & $49(2)$ & $0(2)$ & $7(2)$ & $2(2)$ \\
\hline$C(17)$ & $39(2)$ & $34(2)$ & $54(2)$ & $6(2)$ & $12(2)$ & $-3(2)$ \\
\hline $\mathrm{C}(18)$ & $96(4)$ & $60(3)$ & $123(5)$ & $-5(3)$ & $70(4)$ & $-13(3)$ \\
\hline$C(21)$ & $48(2)$ & $36(2)$ & $36(2)$ & $6(2)$ & $9(2)$ & $-2(2)$ \\
\hline$C(22)$ & $35(2)$ & $30(2)$ & $34(2)$ & $3(2)$ & $3(2)$ & $-3(2)$ \\
\hline$C(23)$ & $36(2)$ & $39(2)$ & $32(2)$ & $7(2)$ & $0(2)$ & $8(2)$ \\
\hline C (24) & $37(2)$ & $43(2)$ & $30(2)$ & $-3(2)$ & $-4(2)$ & $4(2)$ \\
\hline$C(25)$ & $28(2)$ & $53(2)$ & $42(2)$ & $-8(2)$ & $4(2)$ & $-10(2)$ \\
\hline$C(31)$ & $40(2)$ & $68(3)$ & $59(3)$ & $12(2)$ & $-6(2)$ & $-5(2)$ \\
\hline$C(32)$ & $32(2)$ & $41(2)$ & $39(2)$ & $4(2)$ & $-2(2)$ & $-3(2)$ \\
\hline$C(33)$ & $43(2)$ & $42(2)$ & $32(2)$ & $6(2)$ & $-2(2)$ & $8(2)$ \\
\hline$C(34)$ & $57(2)$ & $34(2)$ & $29(2)$ & $7(2)$ & $5(2)$ & $4(2)$ \\
\hline$C(35)$ & $53(2)$ & $43(2)$ & $33(2)$ & $-2(2)$ & $12(2)$ & $-14(2)$ \\
\hline$C(41)$ & $113(4)$ & $62(3)$ & $54(3)$ & $1(2)$ & $16(3)$ & $-5(3)$ \\
\hline C ( 42$)$ & $46(2)$ & $59(3)$ & $44(2)$ & $-1(2)$ & $1(2)$ & $1(2)$ \\
\hline$C(43)$ & $87(4)$ & $82(4)$ & $69(3)$ & $9(3)$ & $23(3)$ & $-2(3)$ \\
\hline
\end{tabular}


Table S4e. Hydrogen coordinates ( $\mathrm{x}$ 10^4) and isotropic displacement parameters $\left(A^{\wedge} 2 \times 10^{\wedge} 3\right)$ of $\operatorname{trans}-\left[\mathrm{Cr}(\mathrm{hbc})(\mathrm{ONO})_{2}\right] \mathrm{BF}_{4}$. Acetone (IV).

\begin{tabular}{|c|c|c|c|c|}
\hline & $\mathrm{x}$ & Y & $\mathrm{z}$ & $\mathrm{U}(\mathrm{eq})$ \\
\hline $\mathrm{H}(1)$ & 1008 & -25 & 1894 & 43 \\
\hline $\mathrm{H}(2 \mathrm{~A})$ & 5235 & 1269 & 484 & 54 \\
\hline $\mathrm{H}(2 \mathrm{~B})$ & 5781 & 2856 & 592 & 54 \\
\hline $\mathrm{H}(3)$ & 3082 & 1480 & 2329 & 36 \\
\hline $\mathrm{H}(4)$ & 4741 & -106 & 1349 & 38 \\
\hline $\mathrm{H}(5)$ & 2016 & 3105 & 1186 & 38 \\
\hline $\mathrm{H}(5 \mathrm{~A})$ & -1876 & 1646 & 3973 & 222 \\
\hline $\mathrm{H}(6)$ & 3703 & 1550 & 210 & 39 \\
\hline $\mathrm{H}(12)$ & 1640 & 2895 & 3341 & 60 \\
\hline $\mathrm{H}(13)$ & 790 & 2661 & 4126 & 68 \\
\hline H (15) & -1139 & 967 & 3008 & 59 \\
\hline $\mathrm{H}(16)$ & -288 & 1172 & 2232 & 51 \\
\hline $\mathrm{H}(17 \mathrm{~A})$ & 1887 & 2685 & 2353 & 50 \\
\hline $\mathrm{H}(17 \mathrm{~B})$ & 1069 & 3040 & 1919 & 50 \\
\hline $\mathrm{H}(18 \mathrm{~A})$ & -433 & 906 & 4478 & 107 \\
\hline $\mathrm{H}(18 \mathrm{~B})$ & -827 & 2641 & 4362 & 107 \\
\hline $\mathrm{H}(21 \mathrm{~A})$ & 2598 & -1782 & 2824 & 60 \\
\hline $\mathrm{H}(21 \mathrm{~B})$ & 1614 & -1371 & 2739 & 60 \\
\hline $\mathrm{H}(21 \mathrm{C})$ & 2272 & -62 & 3004 & 60 \\
\hline $\mathrm{H}(22)$ & 2326 & -1270 & 1843 & 39 \\
\hline $\mathrm{H}(23 \mathrm{~A})$ & 3886 & -1405 & 2122 & 43 \\
\hline $\mathrm{H}(23 \mathrm{~B})$ & 3898 & -501 & 2737 & 43 \\
\hline $\mathrm{H}(24 \mathrm{~A})$ & 4714 & 1615 & 2382 & 44 \\
\hline $\mathrm{H}(24 \mathrm{~B})$ & 5199 & -37 & 2303 & 44 \\
\hline $\mathrm{H}(25 \mathrm{~A})$ & 5880 & 1569 & 1479 & 49 \\
\hline $\mathrm{H}(25 \mathrm{~B})$ & 5258 & 3038 & 1554 & 49 \\
\hline $\mathrm{H}(31 \mathrm{~A})$ & 428 & 2502 & 1027 & 84 \\
\hline $\mathrm{H}(31 \mathrm{~B})$ & 152 & 669 & 1035 & 84 \\
\hline $\mathrm{H}(31 \mathrm{C})$ & 534 & 1393 & 474 & 84 \\
\hline $\mathrm{H}(32)$ & 1607 & -84 & 982 & 46 \\
\hline $\mathrm{H}(33 \mathrm{~A})$ & 1604 & 3137 & 223 & 47 \\
\hline $\mathrm{H}(33 \mathrm{~B})$ & 2068 & 1470 & 132 & 47 \\
\hline $\mathrm{H}(34 \mathrm{~A})$ & 2925 & 3568 & -192 & 48 \\
\hline $\mathrm{H}(34 \mathrm{~B})$ & 2931 & 4439 & 428 & 48 \\
\hline $\mathrm{H}(35 \mathrm{~A})$ & 4466 & 4307 & 679 & 51 \\
\hline $\mathrm{H}(35 \mathrm{~B})$ & 4564 & 3641 & 38 & 51 \\
\hline $\mathrm{H}(41 \mathrm{~A})$ & 8814 & 3750 & 1061 & 113 \\
\hline $\mathrm{H}(41 \mathrm{~B})$ & 7865 & 4093 & 800 & 113 \\
\hline $\mathrm{H}(41 \mathrm{C})$ & 8607 & 4061 & 378 & 113 \\
\hline $\mathrm{H}(43 \mathrm{~A})$ & 7615 & -93 & 891 & 118 \\
\hline $\mathrm{H}(43 \mathrm{~B})$ & 7178 & 1569 & 1025 & 118 \\
\hline $\mathrm{H}(43 \mathrm{C})$ & 8000 & 993 & 1421 & 118 \\
\hline
\end{tabular}

